J. DIFFERENTIAL GEOMETRY

69 (2005) 75-110

\title{
OPTIMAL RIGIDITY ESTIMATES FOR NEARLY UMBILICAL SURFACES
}

\author{
Camillo De Lellis \& Stefan Müller
}

Dedicated to Hermann Karcher

\begin{abstract}
Let $\Sigma \subset \mathbf{R}^{3}$ be a smooth compact connected surface without boundary and denote by $A$ its second fundamental form. We prove the existence of a universal constant $C$ such that

$$
\inf _{\lambda \in \mathbf{R}}\|A-\lambda \operatorname{Id}\|_{L^{2}(\Sigma)} \leq C\left\|A-\frac{\operatorname{tr} A}{2} \operatorname{Id}\right\|_{L^{2}(\Sigma)} .
$$

Building on this, we also show that, if the right-hand side of $\left(\overline{I_{1}^{\prime}}\right)$ is smaller than a geometric constant, $\Sigma$ is $W^{2,2}-$ close to a round sphere.
\end{abstract}

\section{Introduction}

Let $\Sigma \subset \mathbf{R}^{3}$ be a smooth surface. A point $p$ of $\Sigma$ is called umbilical if the principal curvatures of $\Sigma$ at $p$ are equal. A classical theorem in differential geometry states that if $\Sigma$ is connected and all points of $\Sigma$ are umbilical, then either $\Sigma$ is a subset of a round sphere or it is a subset of a plane. Thus, if $\Sigma$ is a compact surface without boundary, then $\Sigma$ must be a round sphere and therefore, its second fundamental form is a constant multiple of the identity.

In the literature, some quantitative versions of this classical rigidity theorem are available. For instance, in [1. in it is proved that if $\Sigma$ is a closed convex surface and the ratio of its principal curvatures are uniformly close to 1 , then $\Sigma$ is close to a round sphere (see page 493). In [1] $\left.\mathbf{1}_{\mathbf{b}}\right]$, the author proves a similar result replacing the $L^{\infty}$ condition by some integral versions of it. We refer to Chapter 6 of [1; $1 \overline{2}]$ for a survey of this and other results on convex surfaces which are almost umbilical. More recently, in their investigations on the gradient flow of the Willmore functional, in [i, $[\overline{8}]$, the authors show that, without any convexity assumption, if $\left\|A-\frac{\operatorname{tr} A}{2} \operatorname{Id}\right\|_{L^{2}}$ is sufficiently small, then $\Sigma$ flows toward

Received 05/30/2003. 
a round sphere (as usual, we denote by $A$ the second fundamental form of $\Sigma)$.

The main theorem of this paper is the following. Here:

- Id denotes the identity $(1,1)$-tensor and the $(0,2)$-tensor naturally associated to it;

- $\AA$ denotes the traceless part of $A$, i.e., the tensor $A-\frac{\operatorname{tr} A}{2} \mathrm{Id}$;

- id : $\mathbf{S}^{2} \subset \mathbf{R}^{3} \rightarrow \mathbf{R}^{3}$ is the standard isometric embedding of the round sphere.

Theorem 1.1. Let $\Sigma \subset \mathbf{R}^{3}$ denote a smooth compact connected surface without boundary and for convenience normalize the area of $\Sigma$ by $\operatorname{ar}(\Sigma)=4 \pi$. Then,

$$
\|A-\mathrm{Id}\|_{L^{2}(\Sigma)} \leq C\|\AA\|_{L^{2}(\Sigma)},
$$

where $C$ is a universal constant. If in addition $\|A\|_{L^{2}(\Sigma)}^{2} \leq 8 \pi$, then there exists a conformal parameterization $\psi: \mathbf{S}^{2} \rightarrow \Sigma$ and a vector $c_{\Sigma} \in \mathbf{R}^{3}$ such that

$$
\left\|\psi-\left(c_{\Sigma}+\mathrm{id}\right)\right\|_{W^{2,2}\left(\mathbf{S}^{2}\right)} \leq C\|\AA\|_{L^{2}(\Sigma)} .
$$

Note that $(\overline{2} \overline{1})$ is a very natural estimate, since $\|\AA\|_{L^{2}(\Sigma)}$ is scaling invariant. Indeed (i2i) can be easily converted into the following scaleinvariant estimate

$$
\left\|A-r_{\Sigma} \operatorname{Id}\right\|_{L^{2}(\Sigma)} \leq C\|\AA\|_{L^{2}(\Sigma)} \quad \text { where } \quad r_{\Sigma}=\sqrt{\frac{\operatorname{ar}(\Sigma)}{4 \pi}} .
$$

In order to have the second estimate of Theorem ilint, it is sufficient to

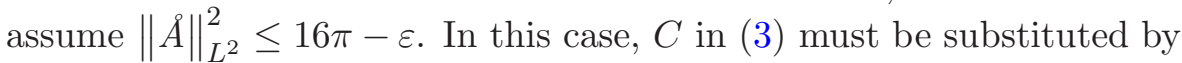
$C(\varepsilon)$, where $C(\varepsilon) \uparrow \infty$ as $\varepsilon \downarrow 0$.

Remark 1.2. Consider the conformal parameterization $\psi$ of Theorem 1.1 . Let us denote by $g$ the metric of $\Sigma$, and by $\sigma$ the standard metric on $\mathbf{S}^{2}$. For the conformal parameterization $\psi: \mathbf{S}^{2} \rightarrow \Sigma$, we have $\psi_{\#} g=h^{2} \sigma$, where the positive smooth function $h$ is the conformal factor of $g$ in the coordinates induced by $\psi$. Then, suitably generalizing

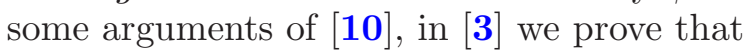

$$
\|h-1\|_{C^{0}} \leq C\|A-\operatorname{Id}\|_{L^{2}(\Sigma)}
$$

for some universal constant $C$.

In Section $\bar{T}_{1}$, we show that these estimates are optimal. More precisely, we construct a sequence of smooth connected compact surfaces $\Sigma_{n}$ without boundary such that 
- $\|\AA\|_{L^{p}} \rightarrow 0$ for every $p<2$;

- $\Sigma_{n}$ converges to the union of two spheres with different radii.

The starting point for proving Theorem $\mathbb{1}_{2} \overline{1}_{-1}$ is the following observation. Let us fix an orthonormal frame $e_{1}, e_{2}$ on $\Sigma$ and denote by $A_{i j}$ the quantities $A\left(e_{i}, e_{j}\right)$ and by $\nabla A_{i j k}$ the quantities $\left[\nabla_{e_{i}} A\right]\left(e_{j}, e_{k}\right)$. The Codazzi equations imply that $\nabla A_{i j k}=\nabla A_{j i k}$. Hence, the symmetry of $A$ gives that $\nabla A$ is a symmetric tensor. In view of this fact, straightforward algebraic computations give that $\nabla_{e_{i}}\left[A_{11}+A_{22}\right]$ can be written as a linear combination of $\nabla_{e_{j}}\left[A_{11}-A_{22}\right]$ and $\nabla_{e_{j}}\left[A_{12}\right]$ plus some error terms of type $A\left(\nabla_{e_{j}} e_{k}, e_{l}\right)$. Moreover, these error terms can be written as non-linear expressions involving $\AA$.

If $A$ were identically 0 , then $\operatorname{tr} A$ would be constant. Roughly speaking, a control on $\AA$ gives some control on the oscillation of $\operatorname{tr} A=$ $A_{11}+A_{22}$. Thus, if $\AA$ is small in a $C^{1}$ sense, then $\Sigma$ would be close to a round sphere. This remark was used in [i];] to give a definition of center of mass for isolated gravitating systems in General Relativity. In view of our result, one should be able to weaken the hypotheses under which Huisken-Yau's construction is possible.

1.1. Structure of the proof. In our case, the difficulties in getting the bound (i2i) are considerably increased by the weakness of the right-hand side of (121) and the non-linearity of the error terms of type $A\left(\nabla_{e_{j}} e_{k}, e_{l}\right)$. The outline of our proof is the following.

- First, we show that, when $\|\AA\|_{L^{2}}$ is sufficiently small, $\Sigma$ is a sphere and there exists a good parameterization by a conformal map $\psi: \mathbf{S}^{2} \rightarrow \Sigma$. By "good", we mean that, after a suitable rescaling, the conformal factor $h$ satisfies uniform $L^{\infty}$ and $W^{1,2}$ bounds (independent of $\Sigma$ ). In order to get these bounds, we derive Hardy

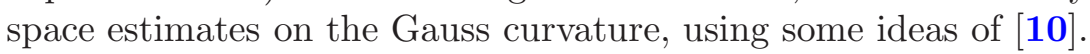
This is accomplished in Section

- We then perform the computations outlined above in the coordinate charts naturally induced by $\psi$. The control on $\psi$ is sufficient to get an $L^{1}$ bound on the non-linear error terms. We use this bound and the regularity theory for the Laplacian to prove the existence of a universal constant $C$ such that

$$
\min _{\lambda \in \mathbf{R}}\|\operatorname{tr} A-\lambda\|_{L^{2, \infty}(\Sigma)} \leq C\|A\|_{L^{2}(\Sigma)},
$$

where $L^{2, \infty}$ is the weak Marcinkiewicz space (see Appendix $\bar{B}^{\prime}$ for the precise definition). This estimate is proved in Proposition

- In Section to the desired stronger estimate (i2i) . This improvement heavily relies on some algebraic computations which exploit the special 
structure of the tensor $A$. The proof uses Hardy space estimates for skew-symmetric quantities and the duality between the Hardy space $\mathcal{H}^{1}$ and BMO.

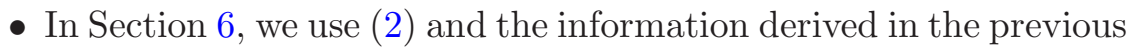
sections to prove the existence of a conformal parameterization $\psi$ which satisfies ( $(\bar{i} i)$. The main difficulty here is due to the action of the conformal group of $\mathbf{S}^{2}$. The existence of $\psi$ is proved into two steps: In the first one, we prove that there is a conformal parameterization with conformal factor $W^{1,2}$-close to 1 ; In the second step, we use the formalism of moving frames to show that this map is $W^{2,2}$-close to a smooth isometric embedding of the standard sphere.

\section{Preliminaries}

2.1. Notation. Throughout this paper, we will use the following notational conventions:

$\begin{array}{ll}\mathbf{S}^{2} & \text { standard sphere } \\ \Sigma & \begin{array}{l}\text { compact connected smooth surface in } \mathbf{R}^{3} \\ \text { without boundary }\end{array} \\ T_{p} \Sigma, T \Sigma & \text { tangent space in } p \text {, tangent bundle } \\ \operatorname{ar}(\Sigma), \mathbf{g}(\Sigma) & \text { area of } \Sigma \text {, genus of } \Sigma \\ D_{r}(x), \partial D_{r}(x) & \text { distance disk and distance circle of radius } \\ & r \text { and center } x \text { in } 2 \text { d Riem. manifolds } \\ \mathcal{D}_{1}, \partial \mathcal{D}_{1} & \text { unit disk and unit circle in } \mathbf{R}^{2} \\ g, \sigma & \text { Riemannian metric on } \Sigma, \text { standard metric } \\ & \text { on } \mathbf{S}^{2} \\ \delta_{i j}, A, N & \text { Kronecker symbol, second fundamental } \\ & \text { form, Gauss map } \\ \operatorname{tr} B, \operatorname{det} B,|B|, \text { Id } & \text { trace of } B, \text { determinant, Hilbert-Schmidt } \\ & \text { norm, identity matrix } \\ \kappa_{1}, \kappa_{2}, K_{G} & \text { principal curvatures, Gaussian curvature } \\ \text { Deg }(\Gamma, \Sigma, u) & \text { topological degree of the map } u: \Gamma \rightarrow \Sigma \\ L^{p}, \mathcal{H}^{1}(\Omega) & L^{p} \text { spaces, Hardy space } \\ \Delta_{\Sigma} & \text { Laplace operator on the Riemannian } \\ & \text { manifold } \Sigma\end{array}$

Let $\psi: \Sigma \rightarrow \Gamma$ be an immersion and $g$ a metric on $\Gamma$. Then, we denote by $\psi^{*} g$ the metric on $\Sigma$ which is the pull back of $g$ via $\psi$. That is

$$
\left(\psi^{*} g\right)_{p}(v, w):=g_{\psi(p)}(d \psi(v), d \psi(w)) \quad \text { for every } v, w \in T_{p}(\Sigma)
$$


A system of coordinates on an open set $U \subset \Sigma$ can be regarded as a smooth diffeomorphism $\psi: \mathbf{R}^{2} \supset \Omega \rightarrow U$. Hence, writing the metric in these coordinates is equivalent to calculating the pull-back metric $\psi^{*} g$.

In the rest of this paper, we assume that $\Sigma$ is compact, connected, and without boundary. Moreover, we assume that $\operatorname{ar}(\Sigma)=4 \pi$ and we set

$$
\delta^{2}:=\int_{\Sigma}|\AA|^{2}
$$

We will make a frequent use of some elementary relations between differential geometric quantities, in particular, the identities

$$
|A|^{2}=\kappa_{1}^{2}+\kappa_{2}^{2}-2 \kappa_{1} \kappa_{2}=|A|^{2}-2 \operatorname{det} A=|A|^{2}-2 K_{G},
$$

combined with Gauss-Bonnet Theorem:

(8) $\int_{\Sigma}|A|^{2}=\int_{\Sigma}|\AA|^{2}+2 \int_{\Sigma} K_{G}=\delta^{2}+2 \int_{\Sigma} K_{G}=\delta^{2}+8 \pi(1-\mathbf{g}(\Sigma))$.

Remark 2.1. Note that

$$
\|A-\operatorname{Id}\|_{L^{2}}^{2} \leq 2 \int_{\Sigma}|A|^{2}+2 \operatorname{ar}(\Sigma) .
$$

Since $\mathbf{g}(\Sigma) \geq 0$, by (18) for every $c>0$ there exists $C>0$ such that

$$
\|A-\operatorname{Id}\|_{L^{2}(\Sigma)} \leq C\|\AA\|_{L^{2}(\Sigma)}^{2} \quad \text { for every } \Sigma \text { with } \delta \geq c .
$$

Thus, it suffices to show (2i) for $\delta$ sufficiently small.

2.2. $\Sigma$ is a sphere. In the following lemma, we show that, when $\delta$ is sufficiently small, $\Sigma$ is a sphere. The proof uses well known elementary facts of differential geometry of surfaces. We report it for the reader's convenience.

Lemma 2.2. If $\delta^{2}<16 \pi$, then $\Sigma$ is a sphere.

Proof. Set $\eta:=16 \pi-\delta^{2}$ and note that

(9) $\int_{\Sigma}|\operatorname{det} A| \leq \frac{1}{2} \int_{\Sigma}|A|^{2} \stackrel{\left(\overline{8}_{1}^{\prime}\right)}{=} 8 \pi-\frac{\eta}{2}+4 \pi(1-\mathbf{g}(\Sigma))<4 \pi(3-\mathbf{g}(\Sigma))$.

Hence, $\mathbf{g}(\Sigma)$ is either 0 , 1 , or 2 . Let $N: \Sigma \rightarrow \mathbf{S}^{2}$ be the Gauss map, which to every point $x \in \Sigma$ associates the exterior unit normal to $\Sigma$ in $x$. Since $A=d N$, the area formula gives

$$
\int_{\Sigma}|\operatorname{det} A|=\int_{\mathbf{S}^{2}} \# N^{-1}(\{\xi\}) d \xi .
$$

Note that $N$ is surjective. Indeed, let $\xi \in \mathbf{S}^{2}$ and consider the largest real number $a$ such that the set $\mathrm{Ex}:=\{x \in \Sigma: x \cdot \xi=a\}$ is not empty. For any $y \in \mathrm{Ex}$, we have $N(x)=\xi$. 
This implies that $\# N^{-1}(\{\xi\}) \geq 1$ and hence gives $\int|\operatorname{det} A| \geq 4 \pi$, which thanks to $(\overline{9})$ rules out the possibility $\mathrm{g}(\Sigma)=2$. Moreover, if $\mathbf{g}(\Sigma)=1$ (i.e., if $\Sigma$ were a torus), the degree $\operatorname{Deg}\left(\Sigma, \mathbf{S}^{2}, N\right)$ would necessarily be 0 , which implies $\# N^{-1}(\{\xi\}) \geq 2$. Hence, ( rule out the possibility $\mathbf{g}(\Sigma)=1$. This gives $\mathbf{g}(\Sigma)=0$ and completes the proof.

q.e.d.

\section{Existence of a good conformal parameterization}

In this section, we show that, if $\delta$ is sufficiently small, then the surface $\Sigma$ has a conformal parameterization which enjoys good bounds.

Definition 3.1. Denote by $\sigma$ the metric on the standard sphere $\mathbf{S}^{2}$ and by $g$ the standard metric on $\Sigma$ as submanifold of $\mathbf{R}^{3}$. If $\psi: \mathbf{S}^{2} \rightarrow \Sigma$ is conformal, then $h$ denotes the unique function $h: \mathbf{S}^{2} \rightarrow \mathbf{R}^{+}$with $h^{2} \sigma=\psi^{*} g$.

Proposition 3.2. Let $\delta^{2}<8 \pi$ and set $\eta:=8 \pi-\delta^{2}$. Then, there exists a constant $C(\eta)$ and a conformal parameterization $\psi: \mathbf{S}^{2} \rightarrow \Sigma$ such that

$$
(C(\eta))^{-1} \leq h \leq C(\eta) \quad\|d h\|_{L^{2}} \leq C(\eta) .
$$

A classical theorem (see for example [i $\left.\mathbf{g}_{1}^{\prime}\right)$ implies the existence of conformal parameterizations $\psi: \mathbf{S}^{2} \rightarrow \Sigma$. However, we cannot hope to have the bounds of Proposition for all such $\psi$ (due to the action of the conformal group). The choice of a good $\psi$ is based on the following remark (cf. [10 10] ). If $h=e^{u}$, then

$$
\int_{\mathbf{S}^{2}} e^{2 u}=4 \pi \quad-\Delta_{\mathbf{S}^{2}} u=K e^{2 u}-1,
$$

where $\Delta_{\mathbf{S}^{2}}$ is the Laplace operator on $\mathbf{S}^{2}$ and $K(x)=K_{\Sigma}(\psi(x))$. If we can bound the norm of the right-hand side of $\left(\begin{array}{l}1 \\ 1\end{array} \overline{2}\right)$ in the Hardy space $\mathcal{H}^{1}$, then the proposition follows from the results of Fefferman and Stein [']i]" (for the definition of $\mathcal{H}^{1}$ and for a precise statement of the result of

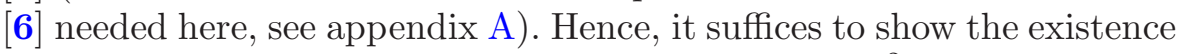
of a constant $C(\eta)$ and of a conformal $\psi$ such that $\left\|K e^{2 u}\right\|_{\mathcal{H}^{1}\left(\mathbf{S}^{2}\right)} \leq C(\eta)$. To derive this estimate, we will use some ideas of [i] $\mathbf{i} \overline{\mathbf{0}}]$ and the following result of [2]:

Theorem 3.3. Let $u \in W^{1, n}\left(\mathbf{R}^{n}, \mathbf{R}^{n}\right)$. Then, there exists a constant $c$ (depending only on $n$ ) such that

$$
\|\operatorname{det} d u\|_{\mathcal{H}^{1}\left(\mathbf{R}^{n}\right)} \leq c\|d u\|_{L^{n}} .
$$


As already pointed out, in order to get the estimates (i $\left.\overline{1}_{1}^{1}\right)$, we have to mod out the action of the conformal group of the sphere. This is accomplished in the following

Lemma 3.4. Assume that $\delta^{2}<8 \pi$ and set $\eta:=8 \pi-\delta^{2}$. Let $x_{1}, x_{2}$, and $x_{3}$ be standard coordinates on $\mathbf{R}^{3}$ and set $\mathbf{S}_{i}^{ \pm}:=\left\{ \pm x_{i}>0\right\} \cap \mathbf{S}^{2}$. Then, there exists a conformal $\psi: \mathbf{S}^{2} \rightarrow \Sigma$ such that

$$
\int_{\psi\left(\mathbf{S}_{i}^{j}\right)}|A|^{2}=8 \pi-\frac{\eta}{2} \quad \text { for all } j \in\{+,-\} \text { and every } i \in\{1,2,3\} .
$$

Proof. Thanks to Lemma $2 \overline{2} . \overline{2}, \Sigma$ is a sphere. Hence, equation (י⿳亠口冋口) implies

$$
\int_{\Sigma}|A|^{2}=16 \pi-\eta
$$

Denote by $e_{i}$ the vectors of the standard basis of $\mathbf{R}^{3}$ relative to the system of coordinates $x_{i}$. For each $i$, we denote by $\mathcal{S}^{i}: \mathbf{S}^{2} \rightarrow \mathbb{C} \cup\{\infty\}$ the stereographic projection which maps $e_{i}$ to the origin and the equator $\left\{x_{i}=0\right\} \cap \mathbf{S}^{2}$ onto the unit circle $\{|z|=1\}$. For each $r>0$, we define $\mathcal{O}_{r}: \mathbb{C} \cup\{\infty\} \rightarrow \mathbb{C} \cup\{\infty\}$ by $\mathcal{O}_{r}(z)=r z$. For every $i \in\{1,2,3\}$ and $r>0$, we denote by $F_{r}^{i}: \mathbf{S}^{2} \rightarrow \mathbf{S}^{2}$ the conformal diffeomorphism $\left(\mathcal{S}^{i}\right)^{-1} \circ \mathcal{O}_{r} \circ \mathcal{S}^{i}$

Choose a conformal parameterization $\varphi: \mathbf{S}^{2} \rightarrow \Sigma$. Note that

$$
\lim _{t \uparrow \infty} \int_{\varphi\left(F_{t}^{1}\left(\mathbf{S}_{1}^{+}\right)\right)}|A|^{2}=\int_{\Sigma}|A|^{2} \quad \text { and } \quad \lim _{t \downarrow 0} \int_{\varphi\left(F_{t}^{1}\left(\mathbf{S}_{1}^{+}\right)\right)}|A|^{2}=0 .
$$

By continuity, there exists a $t$ such that

$$
\int_{\varphi\left(\left(F_{t}^{1}\left(\mathbf{S}_{1}^{+}\right)\right)\right.}|A|^{2}=\frac{1}{2} \int_{\Sigma}|A|^{2}=8 \pi-\frac{\eta}{2} .
$$

Define $\varphi_{1}:=\varphi \circ F_{t}^{1}$ and again note that for some $\tau$, we have

$$
\int_{\varphi_{1}\left(F_{\tau}^{2}\left(\mathbf{S}_{2}^{+}\right)\right)}|A|^{2}=\frac{1}{2} \int_{\Sigma}|A|^{2}=8 \pi-\frac{\eta}{2} .
$$

Note that $F_{\tau}^{2}$ maps $\mathbf{S}_{1}^{+}$onto itself. Thus, we have $\int_{\varphi_{1}\left(F_{\tau}^{2}\left(\mathbf{S}_{1}^{+}\right)\right)}|A|^{2}=$ $8 \pi-\eta / 2$. A similar choice of $F_{\sigma}^{3}$ shows that $\varphi \circ F_{t}^{1} \circ F_{\tau}^{2} \circ F_{\sigma}^{3}$ has the desired properties. q.e.d.

Below, we adopt the following convention. Let $\alpha$ be a 2 -form on $\Sigma$ (resp. on $\mathbf{S}^{2}$, on $\mathbf{R}^{2}$ ), let $\beta$ be the standard volume form on $\Sigma$ (resp. on $\mathbf{S}^{2}$, on $\mathbf{R}^{2}$, and denote by $f$ the function such that $\alpha=f \beta$. If $H$ is any function space, then we write $\|\alpha\|_{H}$ for $\|f\|_{H}$. When $H=\mathcal{H}^{1}$, i.e., the first Hardy space, the maximal function of $f$ will be sometimes called 
"maximal function of $\alpha$ " (here and in what follows, we assume to have fixed a mollifier $\zeta$ and a finite atlas, see Appendix

Proof of Proposition $\overline{3}$. Fix $\psi$ as in Lemma and let $N: \Sigma \rightarrow \mathbf{S}^{2}$ be the Gauss map. Set $N^{\prime}:=N \circ \psi$ and note that $K^{\prime}:=K e^{2 u}$ is the Jacobian determinant of $d N^{\prime}$.

The proof of the $\mathcal{H}^{1}$ estimate is based on some arguments of Section 3 of [10]. We first fix some notation. We denote by $\omega$ the standard volume form on $\mathbf{S}^{2}$. Then, $K^{\prime} \omega$ is the pull-back of $\omega$ via the map $N^{\prime}$, that is $K^{\prime} \omega=\left(N^{\prime}\right)^{*} \omega$. Moreover, any disk $D_{\rho}(x) \subset \mathbf{S}^{2}$ will be identified with a disk $\mathcal{D}_{\rho^{\prime}}=\mathcal{D}_{\rho^{\prime}}(0)$ in the complex plane via the standard stereographic projection which maps $x$ onto 0 .

We will show that there are constants $r$ and $C(\eta)$ with the following property. For any $x \in \mathbf{S}^{2}$, there exists a map $M: \mathbb{C} \rightarrow \mathbf{S}^{2}$ such that

(i) $M=N^{\prime}$ on $\mathcal{D}_{r^{\prime}}\left(\approx D_{r}(x)\right)$;

(ii) $M$ is constant on $\mathbb{C} \backslash \mathcal{D}_{(2 r)^{\prime}}$;

(iii) $\int_{\mathbb{C}} M^{*} \omega=0$;

(iv) $\left\|M^{*} \omega\right\|_{W^{-1,2}}+\|d M\|_{L^{2}} \leq C(\eta)$.

Step 1. From (i)-(iv) to the $\mathcal{H}^{1}$ bound.

We first prove that the existence of $M$ as the above gives an $\mathcal{H}^{1}$ bound for $\left(N^{\prime}\right)^{*} \omega$. We make the usual identification $\mathbf{S}^{2}=P^{1}(\mathbb{C})$ and denote by $\pi: \mathbb{C}^{2} \supset \mathbf{S}^{3} \rightarrow P^{1}(\mathbb{C})$ the Hopf fibration. Then, Proposition 3.4.3 of [1] implies that $M$ lifts to a map $F: \mathbb{C} \rightarrow \mathbf{S}^{3} \subset \mathbb{C}^{2}$ (that is $M=\pi \circ F$ ) with

$$
\|d F\|_{L^{2}}=\|d M\|_{L^{2}}+\left\|M^{*} \omega\right\|_{W^{-1,2}} .
$$

Note that the existence of liftings is guaranteed by condition (iii) (see for example [i. standard basis of $\mathbb{C}^{2}$, then $2 M^{*} \omega=2 F^{*} \pi^{*} \omega=i d F_{1} \wedge d \bar{F}_{1}+i d F_{2} \wedge d \bar{F}_{2}$. Writing $F_{j}$ as $F_{i}^{r e}+i F_{i}^{i m}$, it is easy to see that $i d F_{1} \wedge d \bar{F}_{1}+i d F_{2} \wedge d \bar{F}_{2}$ can be written as linear combination of forms of type $d f_{1} \wedge d f_{2}$, where $d f_{1}, d f_{2} \in L^{2}(\mathbb{C})=L^{2}\left(\mathbf{R}^{2}\right)$. Clearly, $d f_{1} \wedge d f_{2}=(\operatorname{det} d f) d x_{1} \wedge d x_{2}$, where $x_{1}, x_{2}$ are standard coordinates in $\mathbf{R}^{2}$. Hence, we can apply Theorem to derive

$$
\left\|M^{*} \omega\right\|_{\mathcal{H}^{1}} \leq C\|d F\|_{L^{2}} \stackrel{\stackrel{\text { (1i7i) }}{=}}{=} C\|d M\|_{L^{2}}+\left\|M^{*} \omega\right\|_{W^{-1,2}} \stackrel{\text { (iv) }}{\leq} C(\eta) .
$$

Let $g$ be the maximal function of $M^{*} \omega$ (in the sense of equation (10 $10 \overline{2}$ )) Then

$$
\|g\|_{L^{1}\left(D_{r / 2}(x)\right)} \leq\|g\|_{L^{1}\left(\mathbf{R}^{2}\right)}=\left\|M^{*} \omega\right\|_{\mathcal{H}^{1}} \leq C(\eta) .
$$

Let $f$ be the maximal function of $\left(N^{\prime}\right)^{*} \omega$. Since $d N^{\prime} \in L^{2}$, clearly, $\operatorname{det} d N^{\prime} \in L^{1}$ and hence, $\left(N^{\prime}\right)^{*} \omega \in L^{1}$. By the definition of maximal 
functions, we have

$$
\|f\|_{L^{1}\left(D_{r / 2}(x)\right)} \leq\|g\|_{L^{1}\left(D_{r / 2}(x)\right)}+C\left\|\left(N^{\prime}\right)^{*} \omega\right\|_{L^{1}},
$$

where the constant $C$ depends only on $r$. Since $\mathbf{S}^{2}$ can be covered with finitely many disks of radius $r / 2$, we find that $\left\|\left(N^{\prime}\right)^{*} \omega\right\|_{\mathcal{H}^{1}\left(\mathbf{S}^{2}\right)}$ is bounded by a constant depending on $\eta$ and $r$.

Step 2. Construction of $M$ and $W^{-1,2}$ estimate.

We now come to the proof of the existence of constants $r$ and $C(\eta)$ which satisfy (i)-(iv) above. We first construct an intermediate function $\zeta: \mathbb{C} \rightarrow \mathbf{S}^{2}$. The constant $r$ is chosen so small that the disk $D_{2 r}(x)$ is contained in one of the half spheres $\mathbf{S}_{i}^{ \pm}$of Lemma

$$
\int_{D_{2 r}(x)}\left|\operatorname{det} d N^{\prime}\right| \leq \frac{1}{2} \int_{\mathbf{S}_{i}^{ \pm}}\left|d N^{\prime}\right|^{2}=4 \pi-\frac{\eta}{4} .
$$

Using the Fubini-Tonelli Theorem, we can find a $\rho \in] r, 2 r[$ such that

$$
\int_{\partial D_{\rho}(x)}\left|d N^{\prime}\right|^{2} \leq \frac{4 \pi}{r}
$$

We identify $D_{\rho}(x)$ with $\mathcal{D}_{\rho^{\prime}} \subset \mathbb{C}$ (using the stereographic projection, see the discussion above) and we define $\zeta: \mathbb{C} \rightarrow \mathbf{S}^{2}$ by setting:

$$
\zeta=N^{\prime} \quad \text { on } \mathcal{D}_{\rho^{\prime}} \quad \text { and } \quad \zeta(z)=N^{\prime}\left(\rho^{\prime} \frac{z}{|z|}\right) \quad \text { on } \mathbb{C} \backslash \mathcal{D}_{\rho^{\prime}} .
$$

Clearly, $\zeta$ satisfies (i). We now show that

(iv) $)^{\prime}\left\|\zeta^{*} \omega\right\|_{W^{-1,2}\left(\mathcal{D}_{\rho^{\prime}+1}\right)}$ and $\|d \zeta\|_{L^{2}\left(\mathcal{D}_{\rho^{\prime}+1}\right)}$ are bounded by a constant $C(\eta)$.

The bound on $\|d \zeta\|_{L^{2}\left(\mathcal{D}_{\rho^{\prime}+1}\right)}$ is given by the fact that $\left\|d N^{\prime}\right\|_{L^{2}\left(\mathbf{S}_{i}^{ \pm}\right)}$is uniformly bounded and by the choice $\left(\overline{2} \overline{2}_{1}^{\prime}\right)$. We retain

$$
\|d \zeta\|_{L^{2}\left(\mathcal{D}_{\rho^{\prime}+1}\right)} \leq C(\eta) .
$$

We now come to the $W^{-1,2}$ bound. Note that

$$
\operatorname{ar}(\zeta(\mathbb{C})) \leq \int_{D_{2 r}(x)}\left|\operatorname{det} d N^{\prime}\right| \leq 4 \pi-\frac{\eta}{4}
$$

Thus, $\mathbf{S}^{2} \backslash \zeta(\mathbb{C})$ has area at least $\eta / 4$. This means that we can find a closed set $E \subset \mathbf{S}^{2} \backslash \zeta(\mathbb{C})$, with area $\eta / 8$. Arguing as in the proof of

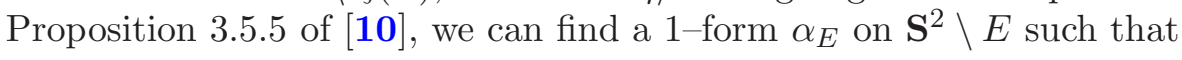

$$
\left\|\alpha_{E}\right\|_{L^{\infty}\left(\mathbf{S}^{2}\right)} \leq \frac{C}{\operatorname{ar}(E)} \quad \text { and } \quad d \alpha_{E}=\omega \quad \text { on } \mathbf{S}^{2} \backslash E,
$$


where $C$ is a universal constant. Using $\alpha_{E}$, one finds $\zeta^{*} \omega=d\left(\zeta^{*} \alpha_{E}\right)$. Let $\varphi \in W^{1,2}\left(\mathcal{D}_{\rho^{\prime}+1}\right)$. Then, since $\zeta$ takes values in $\mathbf{S}^{2} \backslash E$, we have

$$
\int_{\mathcal{D}_{\rho^{\prime}+1}} \varphi \zeta^{*} \omega=\int_{\partial \mathcal{D}_{\rho^{\prime}+1}} \varphi \zeta^{*} \alpha-\int_{\mathcal{D}_{\rho^{\prime}+1}} d \varphi \wedge \zeta^{*} \alpha .
$$

Recall that $\left.\zeta\right|_{\partial \mathcal{D}_{\rho^{\prime}+1}}=\left.N^{\prime}\right|_{\partial D_{\rho^{\prime}}}$. Thus, recalling that $\|\varphi\|_{L^{2}\left(\partial \mathcal{D}_{\rho^{\prime}+1}\right)} \leq$

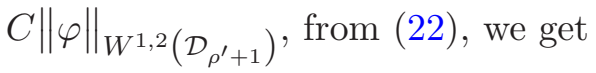

$$
\begin{gathered}
\left|\int_{\partial \mathcal{D}_{\rho^{\prime}+1}} \varphi \zeta^{*} \alpha\right| \leq \frac{C}{\operatorname{ar}(E)}\|\varphi\|_{L^{2}\left(\partial \mathcal{D}_{\rho^{\prime}+1}\right)}\|d \zeta\|_{L^{2}\left(\partial \mathcal{D}_{\rho^{\prime}+1}\right)} \\
\stackrel{\left(200^{\prime}\right)}{=} C(\eta)\|\varphi\|_{W^{1,2}\left(\mathcal{D}_{\rho^{\prime}+1}\right)} .
\end{gathered}
$$

Analogously,

$$
\begin{gathered}
\left|\int_{\mathcal{D}_{\rho^{\prime}+1}} d \varphi \wedge \zeta^{*} \alpha\right| \leq \frac{C}{\operatorname{ar}(E)}\|d \varphi\|_{L^{2}\left(\mathcal{D}_{\rho^{\prime}+1}\right)}\|d \zeta\|_{L^{2}\left(\mathcal{D}_{\rho^{\prime}+1}\right)} \\
\stackrel{\left(21_{1}\right)}{\leq} C\|\varphi\|_{W^{1,2}\left(\mathcal{D}_{\rho^{\prime}+1}\right)}
\end{gathered}
$$

This establishes the $W^{-1,2}$ bound of (iv)'.

Step 3. The existence of $M$.

In this step, we modify $\zeta$ so to reach (ii) and (iii), while keeping (i) and upgrading (iv) ${ }^{\prime}$ to (iv). Consider the restriction of $\zeta$ to $\mathcal{D}_{\rho^{\prime}}$ and define for every regular value $x \in \mathbf{S}^{2}$ its degree $\operatorname{deg}(\zeta, x)$. Standard arguments give that $\operatorname{deg}(\zeta, x)$ is constant on the connected components of $\mathbf{S}^{2} \backslash \zeta\left(\partial \mathcal{D}_{\rho^{\prime}}\right)$. Thus, by continuity, it can be extended to an integer valued piecewise constant function on $\mathbf{S}^{2} \backslash \zeta\left(\partial \mathcal{D}_{\rho^{\prime}}\right)$. Define

$$
U_{0}:=\left\{x \in \mathbf{S}^{2} \mid \operatorname{deg}(\zeta, x)=0\right\} .
$$

Then, $U_{0}$ is an open set contained in $\mathbf{S}^{2} \backslash \zeta\left(\partial \mathcal{D}_{\rho^{\prime}}\right)$. The idea is to choose $y \in U_{0}$ and to take a retraction $R:[0,1] \times \mathbf{S}^{2} \backslash\{y\} \rightarrow \mathbf{S}^{2}$ onto the antipodal of $y$. Then, we define $M=\zeta$ on $\mathcal{D}_{\rho^{\prime}}$ and on $\mathcal{D}_{\rho^{\prime}+1} \backslash \mathcal{D}_{\rho^{\prime}}$ we put

$$
M(z)=R\left(\rho^{\prime}+1-|z|, \zeta(z)\right) .
$$

Since $\zeta\left(\mathcal{D}_{\rho^{\prime}+1} \backslash \mathcal{D}_{\rho^{\prime}}\right)=\zeta\left(\partial \mathcal{D}_{\rho^{\prime}}\right)$, we have $U_{0} \cap \zeta\left(\mathcal{D}_{\rho^{\prime}+1} \backslash \mathcal{D}_{\rho^{\prime}}\right)=\emptyset$. Thus, $M$ is well defined. From the definition of $\left(\underline{2} \overline{3}_{1}^{\prime \prime}\right)$, we clearly have $\operatorname{deg}\left(\mathbb{C}, \mathbf{S}^{2}, M\right) \equiv 0$, and thus $M$ satisfies (iii). Moreover, $\left.M\right|_{\mathcal{D}_{\rho^{\prime}}}=\zeta$ and $\left.M\right|_{\mathbb{C} \backslash \mathcal{D}_{\rho^{\prime}+1}}$ is constant; hence, $M$ satisfies (i) and (ii). The only difficulty is to choose $y$ and the retraction $R$ so as to achieve the bound (iv). 
Clearly, $U_{0}$ contains $\mathbf{S}^{2} \backslash \zeta(\mathbb{C})$ and thus $\operatorname{ar}\left(U_{0}\right) \geq \eta$. Moreover, $U_{0}$ is an open set bounded by a subset of the curve $\gamma=\zeta\left(\partial \mathcal{D}_{\rho}^{\prime}\right)=N^{\prime}\left(\partial D_{\rho}(x)\right)$, which, in view of $\left(\underline{2} \underline{2}_{-1}^{\prime}\right)$ has bounded length. Thanks to Lemma $\bar{C}_{-} \overline{1}_{1}^{\prime}$, there exists a $\delta$, depending on $\operatorname{ar}\left(U_{0}\right)$ and length $(\gamma)$, such that $U_{0}$ contains a ball $D_{\delta}(y)$. Thus, $\delta$ can be chosen bigger than a constant which depends only on $\eta$.

Fix such a $y$ and such a $\delta$ and define a $C^{1}$ map $R:[0,1] \times\left(\mathbf{S}^{2} \backslash\right.$ $\left.D_{\delta}(y)\right) \rightarrow \mathbf{S}^{2}$ which retracts on the antipode $\bar{y}$ of $y$. This can be done so that $\|R\|_{C^{1}}$ depends only on $\eta$. Thus,

$$
\left\|M^{*} \omega\right\|_{W^{-1,2}(\mathbb{C})} \leq C_{1}(\eta)\left\|\zeta^{*} \omega\right\|_{W^{-1,2}\left(D_{\rho^{\prime}+2}(0)\right)} \stackrel{\text { (iv) }^{\prime}}{\leq} C_{2}(\eta) .
$$

An analogous estimate holds for $\|d M\|_{L^{2}}$. This gives (iv) and completes the proof.

q.e.d.

\section{An $L^{2, \infty}$ estimate for $(A-\bar{H} \mathrm{Id})$}

In this section, we prove the following.

Proposition 4.1. There exists $C>0$ such that, if

$$
\operatorname{ar}(\Sigma)=4 \pi, \quad \text { and } \quad \int_{\Sigma}|\AA|^{2} \leq \delta^{2},
$$

then

$$
\left\|A-\left(f_{\Sigma} \frac{\operatorname{tr} A}{2}\right) \operatorname{Id}\right\|_{L^{2, \infty}(\Sigma)} \leq C \delta .
$$

For the definition and properties of the Marcinkiewicz space $L^{2, \infty}$, we refer to Appendix 'B'.'.

Proof. Below, we will prove the existence of a universal constant $C$ such that, for every $\Sigma$ with $\delta^{2} \leq 4 \pi$, there exist two conformal parameterizations $\varphi^{+}, \varphi^{-}: \mathcal{D}_{1} \rightarrow \Sigma$ with the following properties:

(a) $\varphi^{+}\left(\mathcal{D}_{1}\right) \cup \varphi^{-}\left(\mathcal{D}_{1}\right)=\Sigma$;

(b) $\operatorname{ar}\left(\varphi^{+}\left(\mathcal{D}_{1}\right) \cap \varphi^{-}\left(\mathcal{D}_{1}\right)\right) \geq C^{-1}$;

(c) $\left\|\operatorname{tr} A-\lambda^{ \pm}\right\|_{L^{2, \infty}\left(\varphi^{ \pm}\left(\mathcal{D}_{1}\right)\right)} \leq C \delta$ for some constants $\lambda^{ \pm}$.

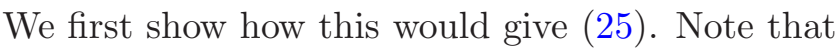

$$
\begin{aligned}
C^{-1}\left|\lambda^{+}-\lambda^{-}\right| & \stackrel{\text { b })}{\leq} \int_{\varphi^{+}\left(\mathcal{D}_{1}\right) \cap \varphi^{-}\left(\mathcal{D}_{1}\right)}\left|\lambda^{+}-\lambda^{-}\right| \\
& \leq \int_{\varphi^{+}\left(\mathcal{D}_{1}\right)}\left|\operatorname{tr} A-\lambda^{+}\right|+\int_{\varphi^{-}\left(\mathcal{D}_{1}\right)}\left|\operatorname{tr} A-\lambda^{-}\right| \\
& \leq C_{1}\left\|\operatorname{tr} A-\lambda^{+}\right\|_{L^{2, \infty}\left(\varphi^{+}\left(\mathcal{D}_{1}\right)\right)}
\end{aligned}
$$




$$
\begin{aligned}
& \quad+C_{1}\left\|\operatorname{tr} A-\lambda^{-}\right\|_{L^{2, \infty}\left(\varphi^{-}\left(\mathcal{D}_{1}\right)\right)} \\
& \stackrel{\text { (c) }}{\leq} 2 C_{1} C \delta .
\end{aligned}
$$

Hence, $\left|\lambda^{+}-\lambda^{-}\right| \leq 2 C_{1} C^{2} \delta$. This means that $\left\|\operatorname{tr} A-\lambda^{+}\right\|_{L^{2, \infty}(\Sigma)} \leq C_{2} \delta$, where $C_{2}$ is another universal constant. Let us set $2 \bar{H}:=\int_{\Sigma} \operatorname{tr} A$. Then,

$$
4 \pi\left|2 \bar{H}-\lambda^{+}\right| \leq \int_{\Sigma}\left|\operatorname{tr} A-\lambda^{+}\right| \leq C_{1}\left\|\operatorname{tr} A-\lambda^{+}\right\|_{L^{2, \infty}(\Sigma)} \leq C_{3} \delta .
$$

This gives $\|\operatorname{tr} A-2 \bar{H}\|_{L^{2, \infty}(\Omega)} \leq C_{4} \delta$. Then,

$$
\|A-\bar{H} \operatorname{Id}\|_{L^{2, \infty}(\Omega)} \leq\left(\int_{\Sigma}|\AA|^{2}\right)^{1 / 2}+\sqrt{2}\left\|\frac{\operatorname{tr} A}{2}-\bar{H}\right\|_{L^{2, \infty}(\Omega)} \leq C_{6} \delta .
$$

Subsections above. To explain the underlying key idea, we have to set some notation. Let $\varphi: \mathcal{D}_{1} \rightarrow \Sigma$ be a conformal parameterization of $\varphi\left(\mathcal{D}_{1}\right)$. We denote by $x_{1}, x_{2}$ a system of orthonormal coordinates in $\mathbf{R}^{2}$. Thus, in these conformal coordinates, the metric of $\Sigma$ is given by $h^{2} \delta_{i j}$. We denote by $e_{i} \in T \Sigma$ the unit vectors $\frac{1}{h} \frac{\partial}{\partial x_{i}}$ and we set $A_{i j}:=A\left(e_{i}, e_{j}\right)$.

Set $f:=\operatorname{tr} A, f_{d}:=A_{11}-A_{22}$, and $f_{m}:=2 A_{12}$. In Subsection $\overline{4} \overline{1}_{1}$, we use the Codazzi-Mainardi equations to control $\nabla f$ in terms of $\bar{f}_{m}, f_{d}$, $\nabla f_{m}$, and $\nabla f_{d}$ (here, if $w: \Sigma \rightarrow \mathbf{R}$, then $\nabla w$ denotes the gradient of $g$ in the Riemannian manifold $\Sigma$; that is, for any vector field $X: \Sigma \rightarrow T \Sigma$, we have $g(\nabla w, X)=d w(X))$.

Potentially, this control will depend in a rather subtle way on the conformal parameterization $\varphi$. This is not a surprise, since the functions $f_{d}$ and $f_{m}$ depend on $\varphi$ (whereas tr $A$ depends only on the immersion of $\Sigma$ in $\mathbf{R}^{3}$ ). In Subsection $\overline{4} .2$, we use the results of Sections $\underline{2}_{1}^{\overline{1}}$ and in order to choose $\varphi^{ \pm}$which satisfy (a) and (b) and enjoy good bounds. We then show that these bounds and the relation derived in Subsection ㄴ. 1 are sufficient to prove (c).

4.1. Key calculation. Let $\varphi, e_{i}, A_{i j}, f, f_{d}$, and $f_{m}$ be as above. When $w$ is a function, $D_{e_{i}} w$ denotes the Lie derivative of $w$ with respect to $e_{i}$, whereas we will use the notations $\partial_{x_{i}} w$ and $w_{i}$ for $\frac{\partial}{\partial x_{i}}[w \circ \varphi]=D_{\frac{\partial}{\partial x_{i}}} w=$ $h D_{e_{i}} w$.

If $X$ is a vector field on $\Sigma$, then we denote by $\nabla_{e_{i}} X$ the covariant derivative of $X$ with respect to $e_{i}$. For every $(2,0)$-tensor $B$ on $\Sigma, \nabla B$ denotes the usual $(3,0)$-tensor given by

$$
\nabla B(X, Y, Z):=D_{X}(B(Y, Z))-B\left(\nabla_{X} Y, Z\right)-B\left(Y, \nabla_{X} Z\right) .
$$


We set $\nabla B_{i j k}=\nabla B\left(e_{i}, e_{j}, e_{k}\right)$ and recall the Codazzi-Mainardi equations:

$$
\nabla A_{i j k}=\nabla A_{j i k} .
$$

To compute $\nabla f$, recall that $\nabla f=\left(D_{e_{1}} f\right) e_{1}+\left(D_{e_{2}} f\right) e_{2}$. Straightforward calculations give

$$
\begin{aligned}
D_{e_{1}} f & =D_{e_{1}}\left(A_{11}+A_{22}\right) \\
& =\nabla A_{111}+\nabla A_{122}+2 A\left(\nabla_{e_{1}} e_{1}, e_{1}\right)+2 A\left(\nabla_{e_{1}} e_{2}, e_{2}\right), \\
D_{e_{1}} f_{d} & =D_{e_{1}}\left(A_{11}-A_{22}\right) \\
& =\nabla A_{111}-\nabla A_{122}+2 A\left(\nabla_{e_{1}} e_{1}, e_{1}\right)-2 A\left(\nabla_{e_{1}} e_{2}, e_{2}\right), \\
D_{e_{2}} f_{m} & =2 D_{e_{2}} A_{12} \\
& =2 \nabla A_{212}+2 A\left(\nabla_{e_{2}} e_{1}, e_{2}\right)+2 A\left(e_{1}, \nabla_{e_{2}} e_{2}\right) .
\end{aligned}
$$

Thus, $D_{e_{1}} f=D_{e_{1}} f_{d}+D_{e_{2}} f_{m}+2 \tilde{R}_{1}$, where

$$
\tilde{R}_{1}=2 A\left(\nabla_{e_{1}} e_{2}, e_{2}\right)-A\left(\nabla_{e_{2}} e_{1}, e_{2}\right)-A\left(e_{1}, \nabla_{e_{2}} e_{2}\right) .
$$

We set $h_{i}:=h D_{e_{1}} h=D_{\frac{\partial}{\partial x_{i}}} h$. Straightforward computations give:

$$
\begin{array}{llrl}
\nabla_{e_{1}} e_{1} & =-\frac{h_{2}}{h^{2}} e_{2} & \nabla_{e_{2}} e_{1}=\frac{h_{1}}{h^{2}} e_{2} \\
\nabla_{e_{1}} e_{2}=\frac{h_{2}}{h^{2}} e_{1} & \nabla_{e_{2}} e_{2}=-\frac{h_{1}}{h^{2}} e_{1} .
\end{array}
$$

Plugging these relations into $\left(\overline{2}_{2} \overline{2} \overline{7}_{1}\right)$, we get

$$
\tilde{R}_{1}:=\frac{2 h_{2}}{h^{2}} A_{12}+\frac{h_{1}}{h^{2}}\left(A_{11}-A_{22}\right)=\frac{h_{2}}{h^{2}} f_{m}+\frac{h_{1}}{h^{2}} f_{d} .
$$

A similar computation for $D_{e_{2}} f$ yields $D_{e_{2}} f=-D_{e_{2}} f_{d}+D_{e_{1}} f_{m}+2 \tilde{R}_{2}$, where $\tilde{R}_{2}$ is given by an expression similar to the one of $\left(\overline{3} \overline{0}_{i}^{i}\right)$. Recall that $h_{i}=D_{\frac{\partial}{\partial x_{i}}} f=\partial_{x_{i}} f$. Hence,

$$
\left\{\begin{array}{l}
\partial_{x_{1}} f=\partial_{x_{1}} f_{d}+\partial_{x_{2}} f_{m}+2 h \tilde{R}_{1} \\
\partial_{x_{2}} f=-\partial_{x_{2}} f_{d}+\partial_{x_{1}} f_{m}+2 h \tilde{R}_{2} .
\end{array}\right.
$$

Denote by $R$ the vector

$$
R:=\left(R_{1}, R_{2}\right):=\left(2 h \tilde{R}_{1}, 2 h \tilde{R}_{2}\right),
$$

by $\operatorname{div}_{E} R$ the "Euclidean" divergence $\partial_{x_{1}} R_{1}+\partial_{x_{2}} R_{2}$ and by $\Delta_{E} f$ the "Euclidean laplacian" $\partial_{x_{1}}^{2} f+\partial_{x_{2}}^{2} f$. Then,

$$
\Delta_{E} f=\partial_{x_{1}}^{2} f_{d}-\partial_{x_{2}}^{2} f_{d}+2 \partial_{x_{1}} \partial_{x_{2}} f_{m}+\operatorname{div}_{E} R .
$$


4.2. Choice of $\varphi^{ \pm}$. Thanks to Lemma 2.2 and Proposition $\overline{2} \cdot 2, \Sigma$ is a sphere and there exist a universal constant $C$ and a conformal parameterization $\psi: \mathbf{S}^{2} \rightarrow \Sigma$ such that

$$
\psi^{*} g=\bar{h}^{2} \sigma \quad C^{-1} \leq \bar{h} \leq C \quad\|d \bar{h}\|_{L^{2}} \leq C .
$$

Clearly, there exist a universal constant $C_{1}$ and two conformal parameterizations $\varphi_{1}, \varphi_{2}: \mathbf{R}^{2} \rightarrow \mathbf{S}^{2}$ such that

$\left(\mathrm{a}^{\prime}\right) \varphi_{1}\left(\mathcal{D}_{1}\right) \cup \varphi_{2}\left(\mathcal{D}_{1}\right)=\mathbf{S}^{2}$

$\left(\mathrm{b}^{\prime}\right) \operatorname{ar}\left(\varphi_{1}\left(\mathcal{D}_{1}\right) \cap \varphi_{2}\left(\mathcal{D}_{1}\right)\right) \geq 1$;

$\left(\mathrm{c}^{\prime}\right)\left\|\varphi_{i}\right\|_{C^{0}(K)}+\left\|\varphi_{i}\right\|_{C^{1}(K)}+\left\|\varphi_{i}\right\|_{C^{2}(K)} \leq C_{1}(K)$ for every compact set $K$.

Let us define $\varphi^{+}:=\psi \circ \varphi_{1}$ and $\varphi^{-}:=\psi \circ \varphi_{2}$. Clearly, $\varphi^{ \pm}$are conformal and for some universal constant $C$, they satisfy (a) and (b). It remains to show (c). Without loss of generality, we show it for $\varphi=\varphi^{+}$. We fix a system of orthonormal coordinates $x_{1}, x_{2}$ in $\mathbf{R}^{2} \supset \mathcal{D}_{1}$ and we adopt the notation of Subsection 4 . Thus, in this system of conformal coordinates, the metric $g$ on $\Sigma$ is given by $h^{2} \delta_{i j}$. Set $f:=\operatorname{tr} A$ as in Subsection $\overline{4}$.

Our goal is to bound $\|f-\lambda\|_{L^{2, \infty}\left(\varphi\left(\mathcal{D}_{1}\right)\right)}$ for some $\lambda \in \mathbf{R}$. Since the conformal factor enjoys $L^{\infty}$ estimates from above and from below, this is equivalent to show that $\|f-\lambda\|_{L^{2, \infty}\left(\mathcal{D}_{1}\right)} \leq C \delta$. Thus, from now on we work in the Euclidean disk $\mathcal{D}_{1}$ : in order to achieve our estimate, we use equation $(\underline{1} \overline{3} \overline{3}-1)$.

First estimate. Let us denote by $\hat{w}$ the Fourier transform of $w$ and by $\check{w}$ the inverse Fourier transform. Moreover, let $\xi$ be the frequency variables. Recall that since $\varphi: \mathbf{R}^{2} \rightarrow \mathbf{S}^{2}$, the functions $f, f_{m}$ and $f_{d}$ are defined everywhere on $\mathbf{R}^{2}$. Let $\zeta$ be a smooth cut-off function supported on $\mathcal{D}_{3 / 2}$ and such that $\varphi=1$ on $\mathcal{D}_{1}$. Define $f^{\prime}$ as

$$
f_{1}:=\frac{\left(\xi_{1}^{2}-\xi_{2}^{2}\right)}{|\xi|^{2}} \widehat{\zeta f}_{d}+2 \frac{\xi_{1} \xi_{2}}{|\xi|^{2}} \widehat{\zeta f}_{m} \quad f^{\prime}:=\check{f}_{1} .
$$

By Plancherel theorem, there exists a constant $C$ (which depends on the cut-off function $\varphi$ ) such that

$$
\left\|f^{\prime}\right\|_{L^{2}} \leq C\left(\left\|f_{d}\right\|_{L^{2}\left(\mathcal{D}_{2}\right)}+\left\|f_{m}\right\|_{L^{2}\left(\mathcal{D}_{2}\right)}\right) \leq C_{1} \delta .
$$

Moreover, on the set $\mathcal{D}_{3 / 2}$, we have

$$
\Delta_{E} f^{\prime}=\partial_{x_{1}}^{2} f_{d}-\partial_{x_{2}}^{2} f_{d}+2 \partial_{x_{1}} \partial_{x_{2}} f_{m}
$$


Second estimate. Let $K(x)=\frac{1}{2 \pi} \log (|x|)$ be the fundamental solution of the Laplacian in $\mathbf{R}^{2}$ and set $f^{\prime \prime}=K * \operatorname{div}_{\mathrm{E}} \mathrm{R}$. Thus, $f^{\prime \prime}=\left(\partial_{x_{1}} K\right) *$ $R_{1}+\left(\partial_{x_{2}} K\right) * R_{2}$. Recall the definition of $R$ in $\left(\overline{3}_{\mathbf{2}} \overline{2}\right)$. By $\left(\overline{3} \overline{0}_{1}^{\prime}\right)$, we have

$$
R_{1}=+\frac{\bar{h}_{2}}{\bar{h}} f_{m}+\frac{\bar{h}_{1}}{\bar{h}} f_{d}
$$

Hence, the estimate $\left(\overline{\overline{3}} \overline{4}_{-1}^{\prime}\right)$ gives that $\left\|R_{1}\right\|_{L^{1}} \leq C \delta$. An analogous estimate holds for $R_{2}$. The locality of convolution, Lemma $\overline{\mathrm{B}} . \overline{1}_{1}^{\prime}$ and Lemma

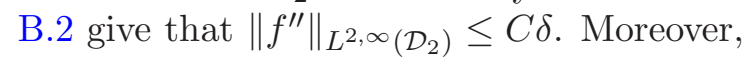

$$
\Delta_{E} f^{\prime \prime}=\operatorname{div} R
$$

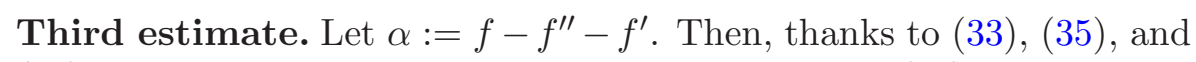

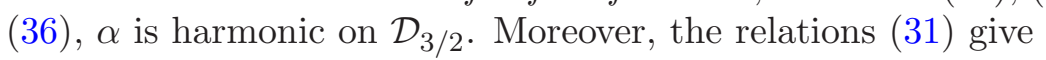

$$
\left\{\begin{array}{l}
\partial_{x_{1}} \alpha=\partial_{x_{1}} f_{d}+\partial_{x_{2}} f_{m}+R_{1}-\partial_{x_{1}}\left(f^{\prime}+f^{\prime \prime}\right) \\
\partial_{x_{2}} \alpha=-\partial_{x_{2}} f_{d}+\partial_{x_{1}} f_{m}+R_{2}-\partial_{x_{2}}\left(f^{\prime}+f^{\prime \prime}\right)
\end{array} .\right.
$$

Let \|\|$\cdot\|\|_{\mathcal{D}_{3 / 2}}$ be a norm which is controlled by both the $L^{1}\left(\mathcal{D}_{3 / 2}\right)$ norm and the $W_{0}^{-1,2}\left(\mathcal{D}_{3 / 2}\right)$ norm. Then, the various estimates give that $\|\mid \nabla \alpha\|_{\mathcal{D}_{3 / 2}} \leq C \delta$. Since $\alpha$ is harmonic and $\mathcal{D}_{1} \subset \subset \mathcal{D}_{3 / 2}$, there is a universal constant $C_{1}$ such that $\|\nabla \alpha\|_{L^{\infty}} \leq C_{1} \delta$. Thus, for some $\lambda>0$ and for some universal constant $C_{2}$, we have $\|\alpha-\lambda\|_{L^{\infty}\left(\mathcal{D}_{1}\right)} \leq C_{2} \delta$. Since $f=f^{\prime}+f^{\prime \prime}+\alpha$, we get

$$
\begin{aligned}
\|f-\lambda\|_{L^{2, \infty}\left(\mathcal{D}_{1}\right)} \leq & C_{3}\left\|f^{\prime}\right\|_{L^{2}\left(\mathcal{D}_{1}\right)}+C_{4}\left\|f^{\prime \prime}\right\|_{L^{2, \infty}\left(\mathcal{D}_{1}\right)} \\
& +C_{5}\|\alpha-\lambda\|_{L^{\infty}\left(\mathcal{D}_{1}\right)} \leq C_{6} \delta .
\end{aligned}
$$

\section{Proof of the $L^{2}$ estimate for $A-\mathrm{Id}$}

In the previous section, we have achieved the following: If we define $2 \bar{H}:=\int_{\Sigma} \operatorname{tr} A$, then $\|A-\bar{H} \operatorname{Id}\|_{L^{2, \infty}} \leq C \delta$. The goal of this section is to use this information to prove

$$
\int_{\Sigma}|A-\mathrm{Id}|^{2} \leq C \delta^{2}
$$

In order to do this, we will show that $\left|1-\bar{H}^{2}\right| \leq C \delta^{2}$. This is sufficient to get $(\overline{3} \overline{8})$. Indeed

$$
\begin{aligned}
|\operatorname{tr} A-2 \bar{H}|^{2} & =\kappa_{1}^{2}+\kappa_{2}^{2}+4 \bar{H}^{2}+2 \kappa_{1} \kappa_{2}-4 \bar{H} \kappa_{1}-4 \bar{H} \kappa_{2} \\
& =\left|\kappa_{1}-\kappa_{2}\right|^{2}+4 \bar{H}^{2}-4 \bar{H} \operatorname{tr} A+4 \operatorname{det} A
\end{aligned}
$$


Integrating $\left(\bar{\beta} \overline{2}_{-1}^{\prime}\right)$ and taking into account $\int_{\Sigma} \operatorname{det} A=4 \pi=\operatorname{ar}(\Sigma)$ and $\int_{\Sigma} \operatorname{tr} A=2 \bar{H} \operatorname{ar}(\Sigma)$, we have

$$
\int_{\Sigma}|\operatorname{tr} A-2 \bar{H} \mathrm{Id}|^{2}=\frac{1}{2} \int_{\Sigma}|\AA|^{2}+16 \pi\left(1-\bar{H}^{2}\right) .
$$

Thus, $\left|1-\bar{H}^{2}\right| \leq C \delta^{2}$ would imply $\int_{\Sigma}|A-\bar{H} \mathrm{Id}|^{2} \leq C \delta^{2}$. Moreover, for $\delta$ small enough, $\left|1-\bar{H}^{2}\right| \leq C \delta^{2}$ implies $(1-\bar{H})^{2} \leq C \delta^{2}$. Since $|A-\mathrm{Id}|^{2} \leq 2|A-\bar{H} \mathrm{Id}|^{2}+2(1-\bar{H})^{2}$, this would give $\left(\overline{3}_{\mathbf{3}} \overline{\bar{q}^{\prime}}\right)$.

For later purposes, we collect the inequality

$$
\|A-\bar{H} \mathrm{Id}\|_{L^{2}}^{2} \leq C \delta^{2}+C_{1}\left|1-\bar{H}^{2}\right|,
$$

which is a direct consequence of the computations above. Moreover, we will make use of the following generalization of Wente's estimate:

Lemma 5.1. Let $\alpha, \beta, \gamma \in C^{\infty}\left(\mathbf{S}^{2}\right)$. Then, there exists a universal constant $C$ such that

$$
\int_{\mathbf{S}^{2}} \alpha d \beta \wedge d \gamma \leq C\|d \alpha\|_{L^{2, \infty}}\|d \beta\|_{L^{2}}\|d \gamma\|_{L^{2}}
$$

Proof. In local charts, thanks to Theorem $\overline{3} . \overline{3}$, we have the $\mathcal{H}^{1}$ estimate

$$
\|d \beta \wedge d \gamma\|_{\mathcal{H}^{1}\left(\mathcal{D}_{1}\right)} \leq C\|d \beta\|_{L^{2}\left(\mathcal{D}_{1}\right)}\|d \gamma\|_{L^{2}\left(\mathcal{D}_{1}\right)}
$$

in the Euclidean disk $\mathcal{D}_{1}$. A finite covering of $\mathbf{S}^{2}$ with smooth coordinate patches yields

$$
\|d \beta \wedge d \gamma\|_{\mathcal{H}^{1}\left(\mathbf{S}^{2}\right)} \leq C\|d \beta\|_{L^{2}\left(\mathbf{S}^{2}\right)}\|d \gamma\|_{L^{2}\left(\mathbf{S}^{2}\right)}
$$

Denote by $\bar{\alpha}$ the average of $\alpha$ on $\mathbf{S}^{2}$. Recalling that $\int d \beta \wedge d \gamma=0$, we get

$$
\int_{\mathbf{S}^{2}} \alpha d \beta \wedge d \gamma=\int_{\mathbf{S}^{2}}(\alpha-\bar{\alpha}) d \beta \wedge d \gamma
$$

Thus, the duality between $\mathcal{H}^{1}$ and $B M O$ (see Theorem lary ${ }_{1}^{\prime}$ -

$$
\int_{\mathbf{S}^{2}} \alpha d \beta \wedge d \gamma \leq C|\alpha|_{B M O}\|d \beta\|_{L^{2}}\|d \gamma\|_{L^{2}}
$$

Thanks to Lemma B.3. we have $|\alpha|_{B M O} \leq C\|d \alpha\|_{L^{2, \infty}}$.

q.e.d. 
5.1. Setting. Using the Gauss-Bonnet formula and the identity $8 \pi \bar{H}=$ $\int_{\Sigma} \operatorname{tr} A$, we get that

$$
4 \pi\left(1-\bar{H}^{2}\right)=\int_{\Sigma} \operatorname{det} A-\bar{H} \int_{\Sigma} \operatorname{tr} A+\bar{H}^{2} \int_{\Sigma} 1 .
$$

We denote by $N: \Sigma \rightarrow \mathbf{S}^{2} \subset \mathbf{R}^{3}$ the Gauss map. Fix a conformal map $\psi: \mathbf{S}^{2} \rightarrow \Sigma \subset \mathbf{R}^{3}$ satisfying the requirements of Proposition conformal map $\varphi: \mathbf{R}^{2} \supset \mathcal{D}_{1} \rightarrow \mathbf{S}^{2}$. Denote by

- $\Psi: \mathcal{D}_{1} \rightarrow \Sigma \subset \mathbf{R}^{3}$ the conformal map $\psi \circ \varphi$;

- $\tilde{h}^{2}$ and $h^{2}$ the conformal factors of $\Psi$ and $\psi$;

- $M$ and $N^{\prime}$ the maps $N \circ \Psi$ and $N \circ \psi$.

Fix an orthonormal system of coordinates $y_{1}, y_{2}, y_{3}$ on $\mathbf{R}^{3}$ and an orthonormal system $x_{1}, x_{2}$ on $\mathcal{D}_{1}$. If $a$ and $b$ are two vectors of $\mathbf{R}^{3}$, then $a \times b$ denotes the vector of $\mathbf{R}^{3}$ which is the standard vector product of $a$ and $b$.

5.2. Algebraic computations. As a first step, we give some formulae for $\tilde{h}^{2}, \tilde{h}^{2}(\operatorname{det} d N) \circ \Psi$ and $\tilde{h}^{2}(\operatorname{tr} d N) \circ \Psi$.

First Computation. Since $\Psi$ is conformal, we have

$$
\operatorname{det} d \Psi=\left|\Psi_{, x_{1}} \times \Psi_{,_{2}}\right| \text {, }
$$

where $\Psi_{, x_{i}}$ denotes the map $\frac{\partial \Psi}{\partial x_{i}}: \mathcal{D}_{1} \rightarrow \mathbf{R}^{3}$. In equation ( $\left(\overline{4}_{-1}^{4}\right)$, we make a slight abuse of notation. Indeed

- On the left-hand side, we consider $\Psi$ as a map taking values on $\Sigma$. Thus, det $d \Psi$ has the usual meaning, since $d \Psi_{p}$ is a linear map from $T_{p} \mathbf{R}^{2} \rightarrow T_{\Psi(p)} \Sigma$.

- On the right-hand side, we consider $\Psi$ as a map taking values on $\mathbf{R}^{3}$.

We now fix the convention on the wedge product of vectors of $\mathbf{R}^{3}$ in such a way that

$$
M \cdot \Psi_{, x_{1}} \times \Psi_{, x_{2}}=\left|\Psi_{, x_{1}} \times \Psi_{, x_{2}}\right| .
$$

Hence, we can write

$$
\tilde{h}^{2}=M \cdot \Psi_{, x_{1}} \times \Psi_{, x_{2}} .
$$

Second Computation. The normal $M$ is perpendicular to both $M_{x_{1}}$ and $M_{, x_{2}}$. Moreover, the orientation convention which yields ( $\left.14{ }_{1}^{4}\right)$ gives

$$
\operatorname{det} d M:=M \cdot M_{, x_{1}} \times M_{, x_{2}} .
$$

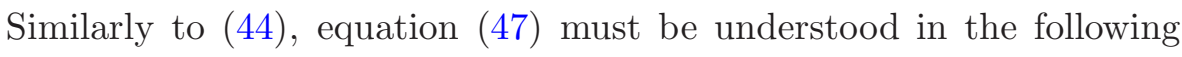
way: 
- On the left-hand side, we consider $M$ as a map taking values on $\mathbf{S}^{2}$. Thus, det $d M$ has the usual meaning;

- On the right-hand side, we consider $M$ as a map taking values on $\mathbf{R}^{3}$.

The discussion above gives the equality

$$
\tilde{h}^{2}(\operatorname{det} d N) \circ \Psi=\operatorname{det} d M=M \cdot M_{, x_{1}} \times M_{, x_{2}} .
$$

Third Computation. Note that $M_{, x_{i}}=[d N \circ \Psi]\left(\Psi_{, x_{i}}\right)$. Thus, thanks to the conformality of $\Psi$, we have

$$
\begin{aligned}
(\operatorname{tr} d N) \circ \Psi= & {\left[d N \circ \Psi\left(\frac{\Psi_{, x_{1}}}{\left|\Psi_{, x_{1}}\right|}\right)\right] \cdot \frac{\Psi_{, x_{1}}}{\left|\Psi_{, x_{1}}\right|} } \\
& +\left[d N \circ \Psi\left(\frac{\Psi_{, x_{2}}}{\left|\Psi_{x_{2}}\right|}\right)\right] \cdot \frac{\Psi_{x_{2}}}{\left|\Psi_{x_{2}}\right|} \\
= & \frac{1}{\tilde{h}^{2}}\left[M_{, x_{1}} \cdot \Psi_{, x_{1}}+M_{x_{2}} \cdot \Psi_{x_{2}}\right] .
\end{aligned}
$$

Since $\Psi$ is conformal, we have

$$
M_{, x_{1}} \cdot \Psi_{, x_{1}}=M_{, x_{1}} \cdot\left(\Psi_{, x_{2}} \times M\right)=M \cdot M_{, x_{1}} \times \Psi_{, x_{2}} .
$$

Thus, we get

$$
\tilde{h}^{2}(\operatorname{tr} d N) \circ \Psi=\left(M \cdot M_{, x_{1}} \times \Psi_{, x_{2}}+M \cdot \Psi_{, x_{1}} \times M_{, x_{2}}\right) .
$$

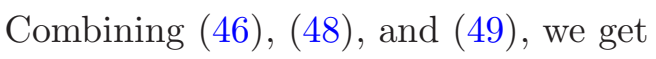

$$
\begin{aligned}
& \int_{\Psi\left(\mathcal{D}_{1}\right)}\left(\operatorname{det} A-\bar{H} \operatorname{tr} A+\bar{H}^{2}\right) \zeta \\
& \quad=\int_{\mathcal{D}_{1}} \tilde{h}^{2}\left((\operatorname{det} d N) \circ \Psi-\bar{H}(\operatorname{tr} d N) \circ \Psi+\bar{H}^{2}\right) \zeta \circ \Psi \\
& =\int_{\mathcal{D}_{1}}\left(M \cdot(M-\bar{H} \Psi)_{, x_{1}} \times(M-\bar{H} \Psi)_{, x_{2}}\right) \zeta \circ \Psi,
\end{aligned}
$$

for every $\zeta \in C_{c}^{\infty}\left(\Psi\left(\mathcal{D}_{1}\right)\right)$.

5.3. Skew-symmetric quantities. Consider two smooth maps $\alpha, \beta$ : $\mathcal{D}_{1} \rightarrow \mathbf{R}^{3}$. Denote by $\alpha_{i}, \beta_{i}, i \in\{1,2,3\}$ the components of $\alpha$ and $\beta$ in a system of orthonormal coordinates of $\mathbf{R}^{3}$. Then, straightforward computations give the following identity:

$$
\left[\alpha \cdot \beta_{, x_{1}} \times \beta_{, x_{2}}\right] d x_{1} \wedge d x_{2}=\sum_{i, j, k=1}^{3} \varepsilon_{i j k} \alpha_{i} d \beta_{j} \wedge d \beta_{k} .
$$


where $\varepsilon_{i j k}$ is the totally antisymmetric tensor given by

$$
\varepsilon_{i j k}=\left\{\begin{aligned}
1 & \text { if }(i, j, k) \text { is an even permutation of }(1,2,3) \\
-1 & \text { if }(i, j, k) \text { is an odd permutation of }(1,2,3) \\
0 & \text { otherwise. }
\end{aligned}\right.
$$

Thus, equations $\left(\underline{5}^{5} \bar{q}_{i}^{i}\right)$ and $(\overline{5} \overline{1} \overline{1})$ give

$$
\begin{aligned}
& \int_{\Psi\left(\mathcal{D}_{1}\right)}\left(\operatorname{det} A-\bar{H} \operatorname{tr} A+\bar{H}^{2}\right) \zeta \\
& \quad=\sum_{i, j, k=1}^{3} \varepsilon_{i j k} \int_{\mathcal{D}_{1}}\left(M_{i} d\left[(M-\bar{H} \Psi)_{j}\right] \wedge d\left[(M-\bar{H} \Psi)_{k}\right]\right) \zeta \circ \Psi
\end{aligned}
$$

for every $\zeta \in C_{c}^{\infty}\left(\Psi\left(D_{1}\right)\right)$. Since $\varphi: \mathcal{D}_{1} \rightarrow \varphi\left(\mathcal{D}_{1}\right) \subset \mathbf{S}^{2}$ is a diffeomorphism, we can use $\varphi^{-1}$ to pull back the forms on the right-hand side of $\left({ }^{5} 2_{2}^{\prime}\right)$ on $\varphi\left(\mathcal{D}_{1}\right)$. Recalling that $N^{\prime}=M \circ \varphi^{-1}$ and $\psi=\Psi \circ \varphi^{-1}$, we get

$$
\begin{aligned}
& \int_{\psi\left(\varphi\left(\mathcal{D}_{1}\right)\right)}\left(\operatorname{det} A-\bar{H} \operatorname{tr} A+\bar{H}^{2}\right) \zeta \\
& \quad=\sum_{i, j, k=1}^{3} \varepsilon_{i j k} \int_{\varphi\left(\mathcal{D}_{1}\right)}\left(N_{i}^{\prime} d\left[\left(N^{\prime}-\bar{H} \psi\right)_{j}\right] \wedge d\left[\left(N^{\prime}-\bar{H} \psi\right)_{k}\right]\right) \zeta \circ \psi .
\end{aligned}
$$

Hence, thanks to the arbitrariness of the conformal map $\varphi$, the previous equation gives that, for every $\zeta \in C^{\infty}\left(\mathbf{S}^{2}\right)$ which is supported in a set of diameter strictly less than $4 \pi$, we have

$$
\begin{aligned}
& \int_{\psi\left(\mathbf{S}^{2}\right)}\left(\operatorname{det} A-\bar{H} \operatorname{tr} A+\bar{H}^{2}\right) \zeta \circ \psi^{-1} \\
& \quad=\sum_{i, j, k=1}^{3} \varepsilon_{i j k} \int_{\mathbf{S}^{2}}\left(N_{i}^{\prime} d\left[\left(N^{\prime}-\bar{H} \psi\right)_{j}\right] \wedge d\left[\left(N^{\prime}-\bar{H} \psi\right)_{k}\right]\right) \zeta .
\end{aligned}
$$

A partition of unity on $\mathbf{S}^{2}$ gives

$$
\begin{aligned}
& \int_{\Sigma}\left(\operatorname{det} A-\bar{H} \operatorname{tr} A+\bar{H}^{2}\right) \\
& =\sum_{i, j, k=1}^{3} \varepsilon_{i j k} \int_{\mathbf{S}^{2}} N_{i}^{\prime} d\left[\left(N^{\prime}-\bar{H} \psi\right)_{j}\right] \wedge d\left[\left(N^{\prime}-\bar{H} \psi\right)_{k}\right] .
\end{aligned}
$$


Integrating by parts, we can write

$$
\begin{aligned}
\int_{\mathbf{S}^{2}} N_{i}^{\prime} d\left[\left(N^{\prime}-\bar{H} \psi\right)_{j}\right] \wedge d\left[\left(N^{\prime}-\bar{H} \psi\right)_{k}\right] \\
\quad=\int_{\mathbf{S}^{2}}-\left(N^{\prime}-\bar{H} \psi\right)_{j} d N_{i}^{\prime} \wedge d\left[\left(N^{\prime}-\bar{H} \psi\right)_{k}\right] .
\end{aligned}
$$

5.4. Final estimates. Thanks to Lemma

$$
\begin{aligned}
& \left|\int_{\mathbf{S}^{2}}\left[\left(N^{\prime}-\bar{H} \psi\right)_{j}\right] d N_{i}^{\prime} \wedge d\left[\left(N^{\prime}-\bar{H} \psi\right)_{k}\right]\right| \\
& \quad \leq\left\|d\left(N^{\prime}-\bar{H} \psi\right)\right\|_{L^{2, \infty}}\left\|d N^{\prime}\right\|_{L^{2}}\left\|d\left(N^{\prime}-\bar{H} \psi\right)\right\|_{L^{2} .}
\end{aligned}
$$

Thus, we conclude that

$$
\begin{aligned}
& \left|\int_{\Sigma}\left(\operatorname{det} A-\bar{H} \operatorname{tr} A+\bar{H}^{2}\right)\right| \\
& \quad \leq C\left\|d N^{\prime}\right\|_{L^{2}\left(\mathbf{S}^{2}\right)}\left\|d\left(N^{\prime}-\bar{H} \psi\right)\right\|_{L^{2}\left(\mathbf{S}^{2}\right)}\left\|d\left(N^{\prime}-\bar{H} \psi\right)\right\|_{L^{2, \infty}\left(\mathbf{S}^{2}\right)},
\end{aligned}
$$

for some universal constant $C$. Since $\psi$ is conformal and satisfies the bounds given by Proposition 3.2 , we have that there exist universal constants $C_{1}, C_{2}$ such that

$$
\begin{aligned}
& \left\|d N^{\prime}\right\|_{L^{2}\left(\mathbf{S}^{2}\right)} \leq C_{1}\|d N\|_{L^{2}(\Sigma)} \leq C_{2} \\
& \left\|d\left(N^{\prime}-\bar{H} \psi\right)\right\|_{L^{2}\left(\mathbf{S}^{2}\right)} \leq C_{1}\|d N-\bar{H} \mathrm{Id}\|_{L^{2}(\Sigma)} \\
& \left\|d\left(N^{\prime}-\bar{H} \psi\right)\right\|_{L^{2, \infty}\left(\mathbf{S}^{2}\right)} \leq C_{1}\|d N-\bar{H} \mathrm{Id}\|_{L^{2, \infty}(\Sigma)} \leq C_{2} \delta .
\end{aligned}
$$

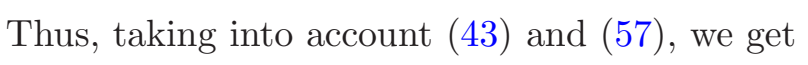

$$
\left|1-\bar{H}^{2}\right| \leq C_{3} \delta\|A-\bar{H} \mathrm{Id}\|_{L^{2}(\Sigma)} .
$$

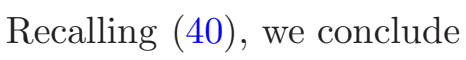

$$
\|A-\bar{H} \mathrm{Id}\|_{L^{2}(\Sigma)}^{2} \leq C \delta^{2}+C_{4} \delta\|A-\bar{H} \mathrm{Id}\|_{L^{2}(\Sigma)}
$$

which, by Young's inequality, yields

$$
\|A-\bar{H} \mathrm{Id}\|_{L^{2}(\Sigma)}^{2} \leq C \delta^{2}+\frac{C_{4}^{2} \delta^{2}}{2}+\frac{\|A-\bar{H} \mathrm{Id}\|_{L^{2}(\Sigma)}^{2}}{2} .
$$

Hence,

$$
\|A-\bar{H} \mathrm{Id}\|_{L^{2}(\Sigma)}^{2} \leq C_{5} \delta^{2}
$$

and plugging this into $\left(\overline{5} \overline{5} \overline{8}_{1}^{\prime}\right)$, we get $\left|1-\bar{H}^{2}\right| \leq C_{6} \delta^{2}$, which completes the proof. 


\section{6. $\Sigma$ is $W^{2,2}$ close to a round sphere}

To complete the proof of Theorem '1. 1 , it remains to show the estimate (13i3), under the assumption that $\left\|\AA_{1}^{3}\right\|_{L^{2}}^{2} \leq 8 \pi$. The difficulties in getting a conformal $\psi$ satisfying $\left(\overline{p_{1}}\right)$ are considerably increased by the action of the conformal group of the sphere. In order to choose $\psi$, as a first step, we impose the normalization conditions of Lemma these conditions imply that the conformal factor of $\bar{\psi}$ is $W^{1,2}$-close to 1 (see Subsection $\left.6.1_{1}^{\prime}\right)$. In a second step, we prove that this, together with the bound on $\|A-\operatorname{Id}\|_{L^{2}\left(D_{\rho}\right)}$ implies that $\psi$ is $W^{2,2}$-close to a smooth

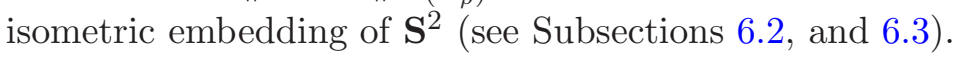

6.1. The conformal factor of $\psi$ is close to 1. Fix $\psi$ as in Lemma 3. goal of this subsection is to show the existence of a universal constant $C$ such that

$$
\left\|e^{u}-1\right\|_{W^{1,2}}+\|u\|_{W^{1,2}} \leq C \delta .
$$

To do so, we first show that for $\delta \downarrow 0$, the map $\psi$ must converge to a conformal map, in fact a rigid motion in view of the normalizations. Then, we use a linearization of the equation $-\Delta_{\mathbf{S}^{2}} u=K e^{2 u}-1$ to get the optimal estimate.

First, we gather all the information acquired in the previous sections

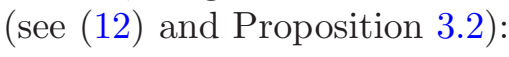

$$
\begin{aligned}
& u \text { satisfies }-\Delta_{\mathbf{S}^{2}} u=K e^{2 u}-1 \text { and } \int e^{2 u}=4 \pi \\
& \|u\|_{L^{\infty}}+\|u\|_{W^{1,2}} \leq C \text { for some universal constant } C \\
& \text { Let } \mathbf{S}_{i}^{ \pm} \text {be as in Lemma } 3 \int_{\mathbf{S}_{i}^{ \pm}}|A|^{2} e^{2 u}=4 \pi+\delta^{2} / 2 \text {. Then, } \int_{\Sigma}|A-\mathrm{Id}|^{2} \leq C \delta^{2} \\
& \int_{\Sigma} \mid{ }^{2 u}
\end{aligned}
$$

Step 1 . We begin by proving the following statement

Fix $p<\infty$ and $\eta>0$. If $\delta>0$ is

$$
\text { sufficiently small, then }\left\|e^{2 u}-1\right\|_{L^{p}}+\|u\|_{L^{p}} \leq \eta \text {. }
$$

Since $e^{2 v}$ is a locally Lipschitz function, thanks to $\left(\begin{array}{l}6 \\ 6\end{array} \overline{1} \overline{1}_{1}^{\prime}\right)$, there exists a constant $C$, independent of $u$, such that

$$
\left|e^{2 u}-1\right| \leq C|u| \text {. }
$$

Thus, we have $\left\|e^{2 u}-1\right\|_{L^{p}} \leq C\|u\|_{L^{p}}$. Assume, by contradiction, that $\left(\overline{6} \overline{4}_{1}^{\prime}\right)$ were false. Then, there exist $\eta>0$ and sequences $\delta_{n} \downarrow 0,\left\{u_{n}\right\} \subset$ $\bar{C}^{\infty}\left(\mathbf{S}^{2}\right)$ such that 
- $e^{u_{n}}$ are the conformal factors of the conformal diffeomorphisms $\psi_{n}: \mathbf{S}^{2} \rightarrow \Sigma_{n} \subset \mathbf{R}^{3}$

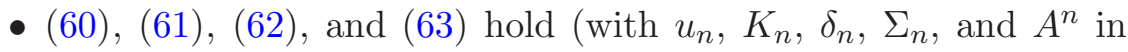
place of $u, K, \delta, \Sigma$, and $A$ );

- $\left\|u_{n}\right\|_{L^{p}} \geq \eta>0$.

Thanks to these assumptions, $\Delta_{\mathbf{S}^{2}} u_{n}$ is a bounded sequence in $L^{1}$. Let $D(\Delta)$ be the set of functions $f \in L^{1}\left(\mathbf{S}^{2}\right)$ with zero average. Recall that $\Delta_{\mathbf{S}^{2}}^{-1}: D(\Delta) \rightarrow W^{1, q}$ is a compact operator for every $q<2$. Thus, a subsequence of $u_{n}$, not relabeled, converges strongly in $W^{1, q}$ to some $u_{\infty}$. Equations $\left(\overline{6}^{\prime}\right)$ and $(\overline{6} \overline{2})$ give that $K_{n}-1$ converges to 0 strongly in $L^{1}$. Since $e^{2 u_{n}}$ is bounded and converges strongly in $L^{q}$ to $e^{2 u_{\infty}}$, by the dominated convergence Theorem, we conclude that $K_{n} e^{2 u_{n}}$ converges

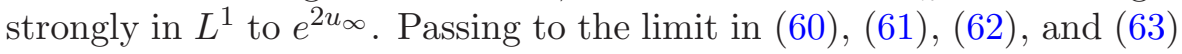
we get

$$
\begin{gathered}
-\Delta_{\mathbf{S}^{2}} u_{\infty}=e^{2 u_{\infty}}-1, \\
\int_{\mathbf{S}_{i}^{ \pm}} e^{2 u_{\infty}}=2 \pi .
\end{gathered}
$$

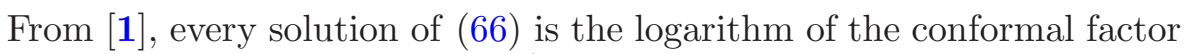
of a conformal diffemorphism $\tilde{\psi}: \mathbf{S}^{2} \rightarrow \mathbf{S}^{2}$. Thus, the normalization

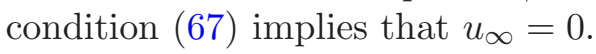

Step 2. Consider the space of functions $\mathcal{S}:=\left\{\|\zeta\|_{\infty} \leq C\right\}$. Then, we claim the existence of a universal constant $C_{1}$ such that

$$
\|\zeta\|_{L^{2}} \leq C_{1}\left(\left\|\Delta_{\mathbf{S}^{2}} \zeta+2 \zeta\right\|_{L^{1}}+\max _{i, j}\left|\int_{\mathbf{S}_{i}^{j}} e^{2 \zeta}-2 \pi\right|\right) \quad \forall \zeta \in \mathcal{S} .
$$

Indeed, set

$$
\eta:=\left\|\Delta_{\mathbf{S}^{2}} \zeta+2 \zeta\right\|_{L^{1}}+\max _{i, j}\left|\int_{\mathbf{S}_{i}^{j}} e^{2 \zeta}-2 \pi\right|
$$

and consider the space

$$
\mathcal{K}:=\left\{\xi \mid-\Delta_{\mathbf{S}^{2}} \xi=2 \xi\right\} .
$$

Note that, if we extend $\xi$ to a 1 -homogeneous function $\bar{\xi}$ on $\mathbf{R}^{3}$, we get that $\bar{\xi}$ is harmonic in $\mathbf{R}^{3} \backslash\{0\}$. Since $\bar{\xi}$ is bounded in every ball, 0 is a removable singularity and $\bar{\xi}$ is an entire harmonic function with linear growth. By the Liouville Theorem, we conclude that $\bar{\xi}$ is a linear function. Thus, $\mathcal{K}$ is the three-dimensional space given by the restriction to $\mathbf{S}^{2}$ of linear functions of $\mathbf{R}^{3}$. 
For $\zeta \in \mathcal{S}$, we denote by $P \zeta$ the $L^{2}$-projection of $\zeta$ on $\mathcal{K}$ and by $P^{\perp} \zeta$ the $L^{2}$-projection on the orthogonal complement of $\mathcal{K}$. Using Sobolev embeddings, it is easy to check that

$$
\left\|P^{\perp} \zeta\right\|_{L^{2}} \leq C_{2} \eta
$$

Since $\mathcal{K}$ has finite dimension, we have

$$
\|P \zeta\|_{\infty} \leq\|\zeta\|_{\infty}
$$

and thus, for some universal constant $C_{3}$, we get

$$
\|P \zeta\|_{\infty}+\left\|P^{\perp} \zeta\right\|_{\infty} \leq C_{3} \quad \forall \zeta \in \mathcal{S} .
$$

Clearly,

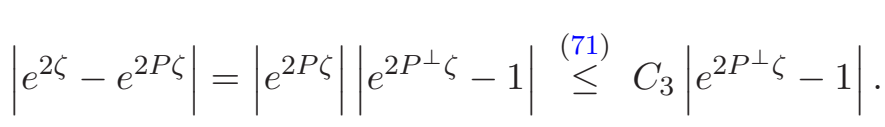

Moreover, since the exponential is a locally Lipschitz function, the bound $\left(\bar{L}_{2}^{1} \overline{1}_{1}^{1}\right)$ gives also

$$
\int_{\mathbf{S}^{2}}\left|e^{2 P^{\perp} \zeta}-1\right| \leq C_{4}\left\|P^{\perp} \zeta\right\|_{L^{1}} \leq C_{5}\left\|P^{\perp} \zeta\right\|_{L^{2}} \stackrel{\left(\begin{array}{c}
70 \\
170
\end{array}\right)}{\leq} C_{6} \eta .
$$

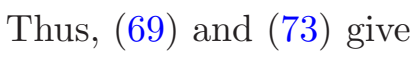

$$
\left|\int_{\mathbf{S}_{i}^{ \pm}} e^{2 P \zeta}-2 \pi\right| \leq C_{7} \eta
$$

Since $P \zeta$ is the restriction of a linear function, it is straightforward to check that

$$
\|P \zeta\|_{L^{2}} \leq C_{8} \eta
$$

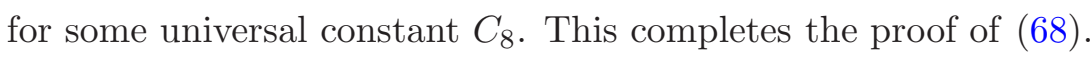

Step 3. We rewrite the first identity of $\left(\overline{6}^{6} \bar{Q}_{i}\right)$ as

$$
-\Delta_{\mathbf{S}^{2}} u-2 u=\left(e^{2 u}-2 u-1\right)+(K-1) e^{2 u} .
$$

Since $\|u\|_{\infty}$ is bounded by a universal constant (see $\left.\left(\overline{6}_{-1}^{6} \overline{1}_{-1}^{i}\right)\right)$, we have

$$
\left\|e^{2 u}-2 u-1\right\|_{L^{1}} \leq C_{1}\|u\|_{L^{2}}^{2}
$$

for some universal constant $C_{1}$. Moreover,

$$
\begin{aligned}
\left\|(K-1) e^{2 u}\right\|_{L^{1}} & =\|\operatorname{det} A-1\|_{L^{1}(\Sigma)} \\
& \leq\|\operatorname{det}(A-\mathrm{Id})\|_{L^{1}(\Sigma)}+\|\operatorname{tr} A-2\|_{L^{1}} \\
& \leq\|A-\operatorname{Id}\|_{L^{2}(\Sigma)}^{2}+C_{2}\|A-\operatorname{Id}\|_{L^{2}(\Sigma)} \stackrel{(5,33)}{\leq} C \delta,
\end{aligned}
$$




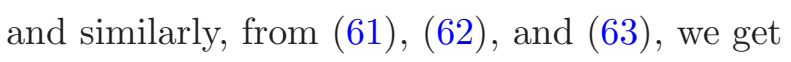

$$
\left|\int_{\mathbf{S}_{i}^{ \pm}} e^{2 u}-2 \pi\right| \leq C \delta .
$$

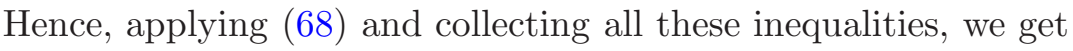

$$
\|u\|_{L^{2}} \leq C_{3}\|u\|_{L^{2}}^{2}+C_{4} \delta .
$$

Thanks to the first step, when $\delta$ is sufficiently small, we have $C_{3}\|u\|_{L^{2}} \leq$ 1/2. Plugging this into (17.

$$
\|u\|_{L^{2}} \leq 2 C_{4} \delta .
$$

Step 4 . We multiply by $u$ the equation

$$
-\Delta_{\mathbf{S}^{2}} u=e^{2 u}-1+(K-1) e^{2 u}
$$

and we integrate by parts to get

$$
\left\|\nabla_{\mathbf{S}^{2}} u\right\|_{L^{2}}^{2} \leq \int_{\mathbf{S}^{2}}|u|\left|e^{2 u}-1\right|+\int_{\mathbf{S}^{2}}|u||\operatorname{det} A-1| e^{2 u}
$$

Notice that

$$
\int|u|\left|e^{2 u}-1\right| \leq C_{1}\|u\|_{L^{2}}^{2} .
$$

Moreover, $|\operatorname{det} A-1| \leq\left|\kappa_{1}-1\right|\left|\kappa_{2}-1\right|+\left|\kappa_{1}+\kappa_{2}-2\right|$, and recalling that $\|u\|_{\infty}$ is uniformly bounded, we get:

$$
\int_{\mathbf{S}^{2}}\left|u\left\|\operatorname{det} A-1 \mid e^{2 u} \leq C_{2}\right\| A-\mathrm{Id}\left\|_{L^{2}(\Sigma)}^{2}+C_{3}\right\| u\left\|_{L^{2}}\right\| A-\mathrm{Id} \|_{L^{2}(\Sigma)} .\right.
$$

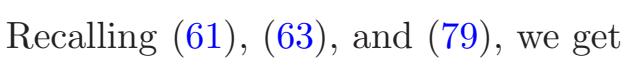

$$
\left\|\nabla_{\mathbf{S}^{2}} u\right\|_{L^{2}}^{2} \leq C_{4} \delta^{2}
$$

which, together with $(\overline{\overline{1}} \overline{\overline{1}} \overline{1})$, gives

$$
\|u\|_{W^{1,2}} \leq C_{5} \delta
$$

Since $\|u\|_{\infty}$ is bounded by a universal constant, the fact that the exponential map is locally Lipschitz gives $\left(\overline{1} \overline{5} \overline{9}_{1}^{\prime}\right)$.

6.2. Cartan formalism. Let $D_{\rho}$ be a disk of $\mathbf{S}^{2}$ and let $\left(e_{1}, e_{2}\right)$ be an orthonormal frame on $D_{\rho}$. We assume that this orthonormal frame is generated by a conformal map $\varphi: \mathcal{D}_{r} \rightarrow D_{\rho}$ via the relations $e_{i}=$ $\partial_{x_{i}} \varphi /\left|\partial_{x_{i}} \varphi\right|$. Moreover, we assume that $\|\varphi\|_{C^{1}}$ is bounded by a universal constant (which is certainly possible if, for instance, $\rho \leq \pi$ ). We define two maps $\Phi, \Psi: D_{\rho} \rightarrow S O(3)$ in the following way

$$
\Phi:=\left(e_{1}, e_{2}, e_{1} \times e_{2}\right) .
$$




$$
\Psi:=\left(e^{-u} d \psi\left(e_{1}\right), e^{-u} d \psi\left(e_{2}\right), e^{-2 u} d \psi\left(e_{1}\right) \times d \psi\left(e_{2}\right)\right) .
$$

Note that $e^{-2 u} d \psi\left(e_{1}\right) \times d \psi\left(e_{2}\right)=N \circ \psi$. Hereby, we fix a system of coordinates in $\mathbf{R}^{3}$ and we regard the elements of $\mathrm{SO}(3)$ as matrices: Thus, according to definition (84) , for $x \in D_{\rho}, \Phi(x)$ is the matrix which has $e_{1}(x), e_{2}(x)$, and $e_{1}(x) \times e_{2}(x)$ as row vectors. We endow $\mathrm{SO}(3)$ with the operator norm and we denote by $B \cdot F$ and by $B^{-1}$ respectively the matrix product of $B$ and $F$, and the inverse of $B$.

We want to show that there exist constants $\rho>0$ and $C>0$ such that

$$
\min _{R \in \mathrm{SO}(3)}\|\Phi-R \cdot \Psi\|_{L^{2}\left(D_{\rho}\right)} \leq C \delta .
$$

Note that the left-hand side of $\left(\overline{8} \overline{6}_{1}^{4}\right)$ is actually independent of the choice of the frame. Thus, though the estimate is derived for the particular frame of $T D_{\rho}$ chosen above, we would conclude:

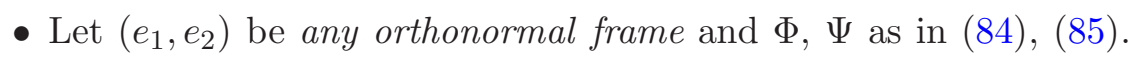
Then $\left(\overline{1}^{1} \overline{6}_{1}^{\prime}\right)$ holds.

An easy covering argument would yield a constant $C^{\prime}$ such that, for some $R \in S O(3)$ :

$$
\text { For every } V \text { and for every frame }\left(e_{1}, e_{2}\right) \text { on } T V \text {, }
$$

$$
\text { we have }\|\Phi-R \cdot \Psi\|_{L^{2}(V)} \leq C^{\prime} \delta \text {. }
$$

One basic property of moving frames (see for instance vol. 3 of [1] 1 ) is the existence of unique 1 -forms with values in skew-symmetric matrices $U$ and $W$ such that

$$
\begin{aligned}
& d \Phi=\Phi \cdot U \\
& d \Psi=\Psi \cdot W .
\end{aligned}
$$

Alternatively, $U$ and $W$ can be regarded as matrices of 1 -forms on $\mathbf{S}^{2}$. We define the norm of $\left|U_{x}\right|$ (for $x \in D_{\rho}$ ) as

$$
\left|U_{x}\right|:=\sup _{v \in T_{x} \mathbf{S}^{2},|v|=1}\left|U_{x}(v)\right|,
$$

where $\left|U_{x}(v)\right|$ is the operator norm of the matrix $U_{x}(v) \in \mathbb{M}^{3 \times 3}$.

We now come to the proof of $\left(\underline{\overline{8}} \overline{\underline{6}} \underline{6}_{i}^{i}\right)$. Consider $\Lambda:=\Phi \cdot \Psi^{-1}$ and compute

$$
\begin{aligned}
d \Lambda & =d \Phi \cdot \Psi^{-1}-\Phi \cdot \Psi^{-1} \cdot d \Psi \cdot \Psi^{-1} \\
& =\Phi \cdot U \cdot \Psi^{-1}-\Phi \cdot \Psi^{-1} \cdot \Psi \cdot W \cdot \Psi^{-1}=\Phi \cdot(U-W) \cdot \Psi^{-1} .
\end{aligned}
$$

The following Lemma is a standard Poincaré inequality (for the reader's

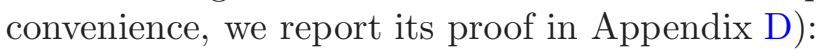


Lemma 6.1. There exists a universal constant $C$ such that for some $R \in S O(3)$, we have

$$
\|\Lambda-R\|_{L^{2}\left(D_{\rho}\right)} \leq C \rho\|d \Lambda\|_{L^{2}\left(D_{\rho}\right)} .
$$

Thus, since $\rho \leq \pi$, there is a constant $C$ such that

$$
\|\Lambda-R\|_{L^{2}\left(D_{\rho}\right)} \leq C\|U-W\|_{L^{2}\left(D_{\rho}\right)} .
$$

To complete the proof of $\left(\overline{8}_{\overline{6}} \overline{6}_{1}\right)$, it is sufficient to show that there is a universal constant $C$ such that

$$
\|U-W\|_{L^{2}\left(D_{\rho}\right)} \leq C \delta .
$$

Let $\theta_{1}, \theta_{2}$ be the basis of the cotangent space $T^{*} M$ which is dual to $\left(e_{1}, e_{2}\right)$. Moreover, recall that

- $e^{v}$ is the conformal factor of $\varphi: \mathcal{D}_{r} \rightarrow D_{\rho}$;

- $x_{1}, x_{2}$ is an orthonormal basis for $\mathcal{D}_{r}$;

- $e_{i}=\partial_{x_{i}} \varphi /\left|\partial_{x_{i}} \varphi\right|=e^{-v} \partial_{x_{i}} \varphi$.

Since the second fundamental form of the sphere is the identity, we have

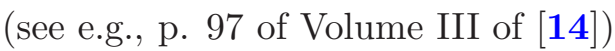

$$
\begin{aligned}
-W_{31}=W_{13}= & A\left(e^{-u} d \psi\left(e_{1}\right), e^{-u} d \psi\left(e_{1}\right)\right) \theta_{1} \\
& +A\left(e^{-u} d \psi\left(e_{1}\right), e^{-u} d \psi\left(e_{2}\right)\right) \theta_{2} \\
-W_{32}=W_{23}= & A\left(e^{-u} d \psi\left(e_{1}\right), e^{-u} d \psi\left(e_{2}\right)\right) \theta_{1} \\
& +A\left(e^{-u} d \psi\left(e_{2}\right), e^{-u} d \psi\left(e_{2}\right)\right) \theta_{2} \\
-U_{31}=U_{13}= & \theta_{1} \\
-U_{32}=U_{23}= & \theta_{2} .
\end{aligned}
$$

Since $\|A-\mathrm{Id}\|_{L^{2}} \leq C \delta$, the previous equations give $\left\|W_{i 3}-U_{i 3}\right\| \leq C \delta$. Thus, it only remains to show that $\left\|U_{12}-W_{12}\right\| \leq C \delta$. Recall that

$$
\begin{aligned}
W_{12}\left(e_{j}\right) & =g\left(\nabla_{e^{-u}}^{\Sigma} d \psi\left(e_{j}\right)\right. \\
U_{12}\left(e_{j}\right) & =\theta^{1}\left(\nabla_{e_{j}}^{\mathbf{S}^{2}} e_{2}\right),
\end{aligned}
$$

where $g$ is the Riemannian metric on $\Sigma$. Thus

$$
\begin{aligned}
U_{12} & =e^{-v}\left\{\left[\partial_{x_{2}} v\right] \theta_{1}-\left[\partial_{x_{1}} v\right] \theta_{2}\right\} \\
W_{12} & =e^{-u \circ \varphi-v}\left\{\left[\partial_{x_{2}}(v+u \circ \varphi)\right] \theta_{1}-\left[\partial_{x_{1}}(v+u \circ \varphi)\right] \theta_{2}\right\} .
\end{aligned}
$$

Recall that $\|\varphi\|_{C^{1}}$ is bounded by a universal constant, that $\left\|e^{-u}-1\right\|_{L^{2}}+$ $\|u\|_{W^{1,2}} \leq C \delta$ and $\|u\|_{\infty} \leq C$. Hence, we conclude that

$$
\left\|U_{12}-W_{12}\right\|_{L^{2}\left(D_{\rho}\right)} \leq C \delta \text {. }
$$


6.3. Conclusion. Let us compose $\psi$ with the inverse of the rotation $R$ appearing in $\left(\overline{8} \bar{z}_{1}\right)$. By abuse of notation, we denote this map by $\psi$ as well. Then, the previous subsection shows the existence of constants $C$ and $\rho$ such that:

- For every disk $D$ of radius $\rho$ in $\mathbf{S}^{2}$ there exists a conformal map $\varphi$ such that $\|\varphi\|_{C^{2}} \leq C$ and, if we define $e_{i}:=\partial_{x_{i}} \varphi /\left|\partial_{x_{i}} \varphi\right|$ and $\Phi, \Psi$

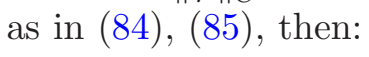

$$
\begin{aligned}
& d \Psi=\Psi \cdot W \quad d \Phi=\Phi \cdot U \\
& \|\Psi-\Phi\|_{L^{2}(D)} \leq C \delta \quad\|U-W\|_{L^{2}(D)} \leq C \delta .
\end{aligned}
$$

Hence, we easily get that

$$
\|d \Psi-d \Phi\|_{L^{2}(D)} \leq\|\Psi \cdot(U-W)\|_{L^{2}(D)}+\|(\Phi-\Psi) \cdot U\|_{L^{2}(D)} \leq C \delta,
$$

where we have also used the fact that $\|U\|_{L^{\infty}}$ depends on $\|\varphi\|_{C^{1}}$, which is bounded by a uniform constant (recall the choice of $\varphi$ ). Denote by id : $\mathbf{S}^{2} \rightarrow \mathbf{R}^{3}$ the standard embedding of the round sphere in the Euclidean space. Note that $(\overline{8} \overline{8} \overline{9})$ gives that $\|d \psi-d(\mathrm{id})\|_{L^{2}(D)} \leq C \delta$. Thus, (since $\rho$ is a fixed constant), by an easy covering argument, we get $\|d \psi-d(i d)\|_{L^{2}\left(\mathbf{S}^{2}\right)} \leq C_{1} \delta$ for some universal constant $C_{1}$. By the Poincaré inequality, there is a vector $c_{\Sigma} \in \mathbf{R}^{3}$ such that

$$
\left\|\psi-\left(c_{\Sigma}+\mathrm{id}\right)\right\|_{W^{1,2}\left(\mathbf{S}^{2}\right)} \leq C_{2} \delta .
$$

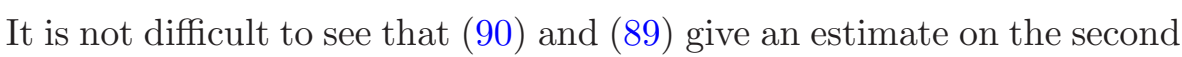
derivatives of $\psi-\left(c_{\Sigma}+\mathrm{id}\right)$, yielding the desired bound

$$
\left\|\psi-\left(c_{\Sigma}+\mathrm{id}\right)\right\|_{W^{2,2}\left(\mathbf{S}^{2}\right)} \leq C_{3} \delta .
$$

Indeed fix a system coordinates on $\mathbf{R}^{3}$ and call $\psi_{k}$, id $\mathrm{id}_{k}$ the components of $\psi$, id. Since $\|\varphi\|_{C^{2}}$ is bounded by a universal constant, it is sufficient to check

$$
\left\|\partial_{x_{i} x_{j}}^{2}\left(\psi_{k}-\mathrm{id}_{k}\right)\right\|_{L^{2}(D)} \leq C_{4} \delta
$$

Note that

$$
\partial_{x_{j}} \psi_{k}=\left|\partial_{x_{j}} \varphi\right|\left[d \psi\left(e_{j}\right)\right]_{k}=h \Psi_{j k}
$$

where $\Psi_{j k}$ denotes the $j k$ entry of the matrix $\Psi$ and $h$ is the conformal factor of $\varphi$. Thus,

$$
\partial_{x_{i} x_{j}}^{2} \psi_{k}=\left(h \partial_{x_{i}} h\right) \Psi_{j k}+h^{2} d \Psi_{j k}\left(e_{i}\right) .
$$

Analogously

$$
\partial_{x_{i} x_{j}}^{2} \operatorname{id}_{k}=\left(h \partial_{x_{i}} h\right) \Phi_{j k}+h^{2} d \Phi_{j k}\left(e_{i}\right) .
$$

Hence, thanks to the uniform bounds on $\|h\|_{L^{\infty}}$ and $\left\|\partial_{x_{j}} h\right\|_{L^{\infty}}$, the

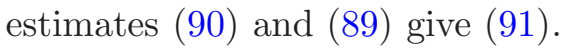




\section{Optimality}

In this section, we prove the optimality of Theorem ili.

Proposition 7.1. There exists a family of smooth connected compact surfaces $\Sigma_{r} \subset \mathbf{R}^{3}$ without boundary such that:

$$
\begin{aligned}
& C \geq \operatorname{ar}\left(\Sigma_{r}\right) \geq c>0 \text { for every } r \\
& \lim _{r \downarrow 0} \int_{\Sigma_{r}}|\AA|^{p}=0 \quad \text { for every } p<2 \\
& \Sigma_{r} \text { converges, in the Hausdorff topology, } \\
& \text { to the union of two round spheres } \\
& \lim _{r \downarrow 0}\left(\inf _{\lambda} \int_{\Sigma_{r}}|A-\lambda \mathrm{Id}|^{p}\right)>0 .
\end{aligned}
$$

Proof. The idea of the construction is the following. Let us take two round spheres $\Sigma_{1}$ and $\Sigma_{2}$ of radii 1 and $1 / 2$. Then, we can glue them with a small hyperbolic neck $\Gamma$ so that the integral $\int_{\Gamma}|A|^{p}$ is as small as we want. We now give the details of this construction. The estimate of the quantity $\int_{\Gamma}|A|^{p}$ will be simplified by using catenoid necks.

Detailed construction. Consider the family of curves $\left\{\gamma_{r}\right\}$ known as catenaries, i.e., the graphs of the functions $f_{r}: \mathbf{R} \rightarrow \mathbf{R}$ given by

$$
f_{r}(x):=r \cosh \left(\frac{x}{r}\right) .
$$

The surface generated by a revolution of $\gamma_{r}$ around the $x$-axis is called a catenoid and will be denoted by $\Gamma_{r}$. It is well known that catenoids are minimal surfaces (see for instance page 202 of [i $\bar{A}^{-}$). Thus, $\operatorname{tr} A=$ $\kappa_{1}+\kappa_{2}=0$ everywhere on $\Gamma_{r}$.

Let $x, y, z$ be a system of coordinates in $\mathbf{R}^{3}$ and assume that the catenoid $\Gamma_{r}$ is given by $|(x, y)|=r \cosh \left(\frac{z}{r}\right)$. For every $r>0$, we take:

- A round sphere of radius $\frac{1}{2}$ centered at a point $\left(0,0, z_{1}\right)$ with $z_{1}>0$ and tangent to $\Gamma_{r}$ in a circle $\gamma_{r}^{1}$.

- A round sphere of radius 1 centered at a point $\left(0,0, z_{2}\right)$ with $z_{2}<0$ and tangent to $\Gamma_{r}$ in a circle $\gamma_{r}^{2}$.

Consider the closed surface $\Sigma_{r}$ which is made of:

- The part of the sphere $\Sigma_{1}$ lying above $\gamma^{1}$ (which we denote by $S_{r}^{2}$ );

- The part of the sphere $\Sigma_{2}$ lying below $\gamma^{2}$ (which we denote by $S_{r}^{1}$ );

- The portion of catenoid lying between $\gamma^{1}$ and $\gamma^{2}$ (which we denote by $T_{r}$ ).

See Fig. 交 below.

Step 1. Behavior of $\Sigma_{r}$ for $r \downarrow 0$. 

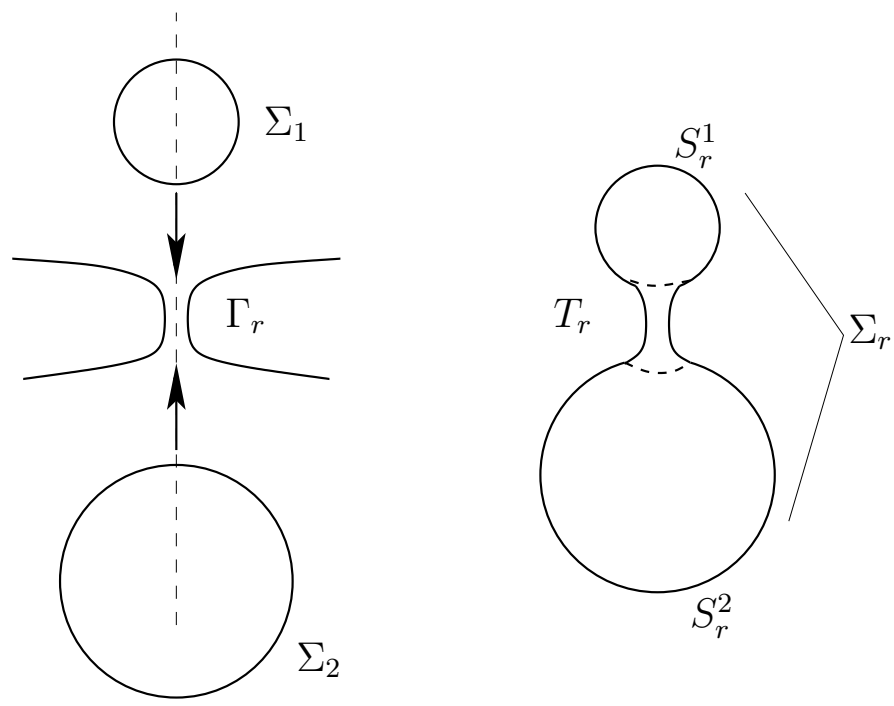

Figure 1. Construction of the surface $\Sigma_{r}$.

The circles $\gamma_{r}^{i}$ are given by

$$
\Gamma_{r} \cap\left\{z=z_{i}(r)\right\}
$$

and straightforward computations give that

$$
\begin{aligned}
& z_{1}(r) \text { is the unique positive solution of } \cosh \left(\frac{z_{1}(r)}{r}\right)=\frac{1}{\sqrt{2 r}} \\
& z_{2}(r) \text { is the unique negative solution of } \cosh \left(\frac{z_{2}(r)}{r}\right)=\frac{1}{\sqrt{r}} .
\end{aligned}
$$

Hence, $z_{i}(r) \downarrow 0$ as $r \downarrow 0$. Moreover, the radius of $\gamma_{r}^{1}$ is $\sqrt{r / 2}$, whereas the radius of $\gamma_{r}^{2}$ is $\sqrt{r}$. Hence, we conclude that

The surfaces $S_{r}^{1}$ and $S_{r}^{2}$ converge, respectively,

(96) to a sphere $S_{\infty}^{1}$ of radius $1 / 2$ and to a sphere $S_{\infty}^{2}$ of radius 1 , which are tangent at $(0,0,0)$.

(97) The area of the neck $T_{r}$ converges to 0.

Step 2. Estimates.

We now prove that

$$
\lim _{r \downarrow 0} \int_{T_{r}}|\AA|^{p}=0
$$


Since $T_{r}$ is a portion of a minimal surface, tr $A=0$ on $T_{r}$. Thus, ( $\left(\overline{9} \bar{g}_{1}^{\prime \prime}\right)$ is equivalent to

$$
\lim _{r \downarrow 0} \int_{T_{r}}|A|^{p}=0 .
$$

Again, because of the minimal surface equation, $2 \operatorname{det} A=-|A|^{2}$ on $T_{r}$. Thus, by Gauss-Bonnet Theorem:

$$
8 \pi=\int_{\Sigma_{r}} 2 \operatorname{det} A=\int_{S_{r}^{1} \cup S_{r}^{2}} 2 \operatorname{det} A-\int_{T_{r}}|A|^{2} .
$$

Since $S_{r}^{1}$ and $S_{r}^{2}$ are both portions of round spheres, we have

$$
\int_{S_{r}^{1} \cup S_{r}^{2}} 2 \operatorname{det} A \leq 16 \pi .
$$

Thus, $\int_{T_{r}}|A|^{2} \leq 8 \pi$ and, by Hölder inequality,

$$
\int_{T_{r}}|A|^{p} \leq\left(\operatorname{ar}\left(T_{r}\right)\right)^{\frac{2-p}{2}}\left(\int_{T_{r}}|A|^{2}\right)^{\frac{p}{2}} \leq(8 \pi)^{\frac{p}{2}}\left(\operatorname{ar}\left(T_{r}\right)\right)^{\frac{2-p}{2}} .
$$

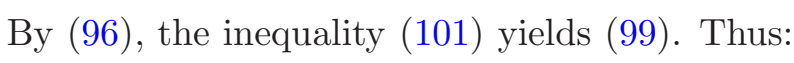

- The bound ( $\overline{9} \overline{2} \overline{2})$ is trivially satisfied.

- Since $S_{r}^{1}$ and $S_{r}^{2}$ are subsets of round spheres, we have

$$
\int_{\Sigma_{r}}|\AA|^{p}=\int_{T_{r}}|\AA|^{p}
$$

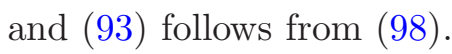

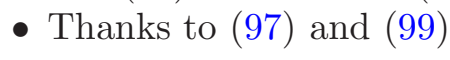

$$
\begin{aligned}
\lim _{r \downarrow 0}\left(\inf _{\lambda} \int_{\Sigma_{r}}|A-\lambda \mathrm{Id}|^{p}\right) & =\inf _{\lambda}\left(\int_{S_{\infty}^{1}}|A-\lambda \mathrm{Id}|^{p}+\int_{S_{\infty}^{2}}|A-\lambda \mathrm{Id}|^{p}\right) \\
& =\inf _{\lambda}\left[2 \pi\left(\frac{1}{2}-\lambda\right)^{2}+8 \pi(1-\lambda)^{2}\right]>0,
\end{aligned}
$$

which gives $\left(\overline{9} \overline{\underline{9}} \overline{5}_{1}^{1}\right)$.

Note that the surfaces just constructed are $C^{1}$ and piecewise $C^{2}$. However, they are all surfaces of revolution: The curves which generate them are $C^{1}$ and piecewise $C^{\infty}$, where the higher derivatives have four points of jump discontinuity. Hence, a standard smoothing argument yields a family of surfaces of revolution which are $C^{\infty}$ and satisfy all the requirements of the Proposition.

q.e.d. 


\section{Appendix A. Hardy and BMO spaces}

We recall here the definitions of Hardy and BMO spaces (see for example [15], sections $1,2,3$ and 4$)$. We fix a $\zeta \in C_{c}^{\infty}\left(\mathbf{R}^{n}\right)$ with $\int \zeta=1$ and we define $\zeta_{\varepsilon}$ as $\zeta_{\varepsilon}(x)=\varepsilon^{-n} \zeta\left(\frac{x}{\varepsilon}\right)$. Then, for every $f \in L_{l o c}^{1}\left(\mathbf{R}^{n}\right)$, we define the maximal function $M_{\zeta} f$ as

$$
M_{\zeta} f(x):=\sup _{r>0}\left|f * \zeta_{r}(x)\right| .
$$

In a similar way, for $M>0$, we define a local maximal function

$$
M_{\zeta}^{M} f(x):=\sup _{M>r>0}\left|f * \zeta_{r}(x)\right| .
$$

Definition A.1. The Hardy space $\mathcal{H}^{1}\left(\mathbf{R}^{n}\right)$ consists of the functions $f \in L_{\text {loc }}^{1}\left(\mathbf{R}^{n}\right)$ such that $M_{\zeta} f \in L^{1}\left(\mathbf{R}^{n}\right)$ for some $\zeta$. Similarly, if $\Omega \subset \mathbf{R}^{n}$, $\mathcal{H}_{\text {loc }}^{1}(\Omega)$ is the subset of $L_{\text {loc }}^{1}$ consisting of those functions $f$ such that $M_{\zeta}^{M} f \in L_{\text {loc }}^{1}$ for some $\zeta$ and some $M$.

Having fixed $\zeta$, we can endow $\mathcal{H}^{1}\left(\mathbf{R}^{n}\right)$ with the norm $\left\|M_{\zeta} f\right\|_{L^{1}\left(\mathbf{R}^{n}\right)}$, thus getting a Banach space (see [i, $\mathbf{1} \mid \bar{j}])$. Different choices of $\zeta$ induce equivalent norms. Moreover, if $f \in \mathcal{H}_{\text {loc }}^{1}(\Omega)$ and $\Phi$ is a diffeomorphism of $\Omega$, then $f \circ \Phi \in \mathcal{H}_{\mathrm{loc}}^{1}(\Omega)$. Hence, using a finite atlas of coordinate patches, it is possible to define $\mathcal{H}^{1}(\Sigma)$ for any compact Riemannian manifold $\Sigma$. Similarly, after fixing a $\zeta$, an $M>0$, and a finite atlas, one can define a local maximal function $M f$ for $f \in \mathcal{H}^{1}(\Sigma)$ and a norm $\|f\|_{\mathcal{H}^{1}(\Sigma)}:=\|M f\|_{L^{1}}$. Different choices induce equivalent norms.

We recall the following celebrated result of ["6in] :

Theorem A.2. Let $w \in \mathcal{H}^{1}\left(\mathbf{R}^{2}\right)$. Then, the equation $\Delta_{\mathbf{R}^{2}} u=w$ admits a continuous solution $u_{0}: \mathbf{R}^{2} \rightarrow \mathbf{R}$ which satisfies

$$
\left\|\nabla^{2} u_{0}\right\|_{L^{1}}+\left\|d u_{0}\right\|_{L^{2}}+\left\|u_{0}\right\|_{L^{\infty}} \leq C\|w\|_{\mathcal{H}^{1}},
$$

for some universal constant $C$.

Using a partition of unity and local coordinate patches, Theorem iA $\mathrm{A}^{-} . \overline{2}$ yields the following

Corollary A.3. Let $w \in \mathcal{H}^{1}\left(\mathbf{S}^{2}\right)$. Then, the equation $\Delta_{\mathbf{S}^{2}} u=w$ admits a continuous solution $u_{0}$ which satisfies

$$
\left\|u_{0}\right\|_{W^{2,1}\left(\mathbf{S}^{2}\right)}+\left\|d u_{0}\right\|_{L^{2}\left(\mathbf{S}^{2}\right)}+\left\|u_{0}\right\|_{L^{\infty}} \leq C\|w\|_{\mathcal{H}^{1}\left(\mathbf{S}^{2}\right)} .
$$

Remark A.4. Since harmonic functions on $\mathbf{S}^{2}$ are constant, the general solution of $\Delta_{\mathbf{S}^{2}} u=w$ can be written as $u=u_{0}+c$. Thus, the normalization condition

$$
\int_{\mathbf{S}^{2}} e^{2 u}=4 \pi
$$




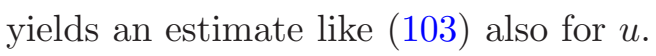

In Section ${ }_{15}^{15}$, we use the duality between BMO and $\mathcal{H}^{1}$, due to Fefferman.

Definition A.5. Let $f \in L_{l o c}^{1}\left(\mathbf{R}^{n}\right)$. We say that $f \in B M O$ if

$$
|f|_{B M O}:=\sup _{x \in \mathbf{R}^{n}} \sup _{r>0} \frac{1}{\left|B_{r}(x)\right|} \int_{B_{r}(x)}\left|f-f^{x, r}\right| \quad \text { is finite, }
$$

where $f^{x, r}$ denotes the average of $f$ on $B_{r}(x)$. We can extend the definition to compact surfaces by taking the second supremum among disks of radius smaller than the diameter of $\Sigma$.

Theorem A.6. Let $f, w \in C_{c}^{\infty}\left(\mathbf{R}^{n}\right)$. Then,

$$
\left|\int f w\right| \leq C_{\zeta}\|f\|_{\mathcal{H}^{1}}|w|_{B M O}
$$

where $C_{\zeta}$ depends only on the kernel $\zeta \in C_{c}^{\infty}\left(\mathbf{R}^{n}\right)$ which defines $\|f\|_{\mathcal{H}^{1}}=$ $\left\|M_{\zeta} f\right\|_{L^{1}}$

Again, using local charts and a partition of unity, we get

Corollary A.7. Let $f, w \in C^{\infty}\left(\mathbf{S}^{2}\right)$. Then, there exists a constant $C$ (depending only on the choices involved in the definition of $\|f\|_{\mathcal{H}^{1}\left(\mathbf{S}^{2}\right)}$ ) such that

$$
\left|\int_{\mathbf{S}^{2}} f w\right| \leq C\|f\|_{\mathcal{H}^{1}\left(\mathbf{S}^{2}\right)}\left[|w|_{B M O\left(\mathbf{S}^{2}\right)}+\left|f_{\mathbf{S}^{2}} w\right|\right]
$$

\section{Appendix B. The space $L^{2, \infty}$}

Given a measure space $\Omega$ with a $\sigma$-finite measure $\mu$, the Marcinkiewicz space $L^{2, \infty}(\Omega, \mu)$ is defined as the set of functions

$$
\left\{f \mid \text { there exists } C>0: \mu\left(\left\{f^{2} \geq k\right\}\right) \leq \frac{C}{k} \text { for every } k>0\right\} .
$$

For every $f \in L^{2, \infty}$, it is natural to define

$$
|f|_{L^{2, \infty}}:=\inf \left\{C: \mu\left(\left\{f^{2} \geq k\right\}\right) \leq \frac{C}{k} \text { for every } k>0\right\} .
$$

$|\cdot|$ is not a norm. However, it is possible to define a norm $\|\cdot\|_{L^{2, \infty}}$ which endows $L^{2, \infty}$ of a Banach space structure and such that

$$
\frac{1}{k}\|\cdot\|_{L^{2, \infty}} \leq|\cdot|_{L^{2, \infty}} \leq k\|\cdot\|_{L^{2, \infty}},
$$

see e.g. Section 1.8 of [i] the following two lemmas: 
Lemma B.1. If $f \in L^{2, \infty}\left(\mathbf{R}^{n}\right), w \in L^{1}\left(\mathbf{R}^{n}\right)$, then

$$
\|f * w\|_{L^{2, \infty}} \leq\|f\|_{L^{2, \infty}}\|w\|_{L^{1}} .
$$

Lemma B.2. Let $K$ be the fundamental solution of the Laplacian in $\mathbf{R}^{2}$ given by $K(x)=\frac{1}{2 \pi} \log (|x|)$. Then, $\nabla K \in L^{2, \infty}(U)$ for every bounded open set $U \subset \mathbf{R}^{2}$.

Lemma $\bar{B}$. 1 , follows easily from the fact that $\|\cdot\|_{L^{2, \infty}}$ is a norm, while Lemma ${ }^{1} .2-1$ is obtained directly from the definition of $|\cdot|_{L^{2, \infty}}$. Finally, in the proof of Theorem 1, 1, we need the following

Lemma B.3. Let $u \in C^{\infty}\left(\mathbf{S}^{2}, \mathbf{R}\right)$. Then, there exists a universal constant $C$ such that

$$
|u|_{B M O\left(\mathbf{S}^{2}\right)} \leq C\|d u\|_{L^{2, \infty}\left(\mathbf{S}^{2}\right)} .
$$

Proof. Lemma $L^{2}\left(\mathbf{S}^{2}\right)$ and the fact that $|u|_{\mathbf{R}^{2}}$ and $|u|_{L^{2, \infty}\left(\mathbf{R}^{2}\right)}$ are both invariant under the rescalings $x \rightarrow r x$. We recall the argument for the reader's convenience.

Using local charts, it suffices to prove

$$
|u|_{B M O\left(\mathcal{D}_{1}\right)} \leq C\|d u\|_{L^{2, \infty}\left(\mathcal{D}_{1}\right)}
$$

where $\mathcal{D}_{1}$ is the Euclidean unit disk. Recall that

$$
|u|_{B M O\left(\mathcal{D}_{1}\right)}:=\sup _{y \in \mathcal{D}_{1}}\left[\sup _{r<\operatorname{dist}\left(y, \partial \mathcal{D}_{1}\right)} \frac{1}{\operatorname{ar}\left(\mathcal{D}_{r}(y)\right)} \int_{\mathcal{D}_{r}(y)}\left|u-u^{y, r}\right|\right],
$$

In view of the definition of $|u|_{B M O\left(\mathcal{D}_{1}\right)}$, it would be sufficient to prove

$$
\frac{1}{\operatorname{ar}\left(\mathcal{D}_{r}(y)\right)} \int_{\mathcal{D}_{r}(y)}\left|u-u^{y, r}\right| \leq C\|d u\|_{L^{2, \infty}\left(\mathcal{D}_{r}(y)\right)} \quad \text { for all } r<1 .
$$

By invariance under translations, we can assume $y=0$. Moreover, we can assume that $r=1$. Indeed, define $u_{r}(x):=u(r x)$. Then,

$$
\frac{1}{\operatorname{ar}\left(\mathcal{D}_{r}\right)} \int_{\mathcal{D}_{r}}\left|u-u^{0, r}\right|=\frac{1}{\operatorname{ar}\left(\mathcal{D}_{1}\right)} \int_{\mathcal{D}_{1}}\left|u_{r}-u_{r}^{0,1}\right|
$$

and

$$
\|u\|_{L^{2, \infty}\left(\mathcal{D}_{r}\right)} \leq k|u|_{L^{2, \infty}\left(\mathcal{D}_{r}\right)}=k\left|u_{r}\right|_{L^{2, \infty}\left(\mathcal{D}_{1}\right)} \leq k^{2}\left\|u_{r}\right\|_{L^{2, \infty}\left(\mathcal{D}_{1}\right)} .
$$

Thus, the proof reduces to the inequality

$$
\int_{\mathcal{D}_{1}}\left|u-u^{0,1}\right| \leq C\|d u\|_{L^{2, \infty}\left(\mathcal{D}_{1}\right)} .
$$

Clearly, for some universal constant $C$, we have

$$
\|d u\|_{L^{1}\left(\mathcal{D}_{1}\right)} \leq C\|d u\|_{L^{2, \infty}\left(\mathcal{D}_{1}\right)} .
$$


Moreover, the Poincaré and Schwartz inequalities give

$$
\begin{aligned}
\int_{\mathcal{D}_{1}}\left|u-u^{0,1}\right| & \leq \pi^{1 / 2}\left\|u-u^{0,1}\right\|_{L^{2}\left(\mathcal{D}_{1}\right)} \\
& \leq C_{1} \pi^{1 / 2}\|d u\|_{L^{1}\left(\mathcal{D}_{1}\right)} \leq C_{1} C \pi^{1 / 2}\|d u\|_{L^{2, \infty}\left(\mathcal{D}_{1}\right)} .
\end{aligned}
$$

This completes the proof.

q.e.d.

\section{Appendix C. Lemma on open sets}

Lemma C.1. Let $U \subset \mathbf{S}^{2}$ be an open set and assume that $\partial U \subset \gamma$, where $\gamma$ is a closed curve. Then, there exists a constant $\delta>0$, depending only on $\operatorname{ar}(U)$ and len $(\gamma)$ such that $U$ contains an open disk of radius $\delta$.

Proof. We argue by contradiction. Then, there exist a sequence of open sets $U_{n}$ and a sequence of closed curves $\gamma_{n}$ such that:

1) $\lim _{n} \operatorname{len}\left(\gamma_{n}\right)=C_{1}>0$ and $\lim _{n} \operatorname{ar}\left(U_{n}\right)=C_{2}>0$;

2) For every $\delta>0$, there exists $N$ such that, for every $n>N, U_{n}$ does not contain any disk of radius $\delta$.

Let us parameterize $\gamma_{n}$ by arc-length. Then, there is a subsequence, not relabeled, which converges uniformly to a Lipschitz curve $\gamma_{\infty}$. Hence, up to subsequences, $\bar{U}_{n}$ converges, in the Hausdorff topology, to a closed set $\bar{U}_{\infty}$ whose boundary is contained in $\gamma_{\infty}$. Due to 2 ., the set $\bar{U}_{\infty}$ has empty interior and thus $\operatorname{ar}\left(\bar{U}_{\infty}\right)=\operatorname{ar}\left(\partial \bar{U}_{\infty}\right)=0$. But 1. implies that $\operatorname{ar}\left(\bar{U}_{\infty}\right)=C_{2}>0$. This is the desired contradiction. $\quad$ q.e.d.

\section{Appendix D. Poincaré inequality for $\mathrm{SO}(3)$-valued maps}

Here, we give a proof of Lemma ${ }^{1}, 1^{\prime}$. We embed $S O(3) \subset \mathbb{M}^{3 \times 3}=\mathbf{R}^{9}$ and we set

$$
\bar{\Lambda}=\frac{1}{\operatorname{ar}\left(D_{\rho}\right)} \int_{D_{\rho}} \Lambda
$$

Since the operator norm on $\mathbb{M}^{3 \times 3}$ is equivalent to the Euclidean norm on $\mathbf{R}^{9}$, the Poincaré inequality yields a constant $C$ such that

$$
\|\Lambda-\bar{\Lambda}\|_{L^{2}\left(D_{\rho}\right)} \leq C \rho\|d \Lambda\|_{L^{2}(D \rho)} .
$$


Note that

$$
\begin{aligned}
\operatorname{dist}(\bar{\Lambda}, \mathrm{SO}(3))^{2} & =\frac{1}{\operatorname{ar}\left(D_{\rho}\right)} \int_{D_{\rho}} \operatorname{dist}(\bar{\Lambda}, S O(3))^{2} \\
& \leq \frac{1}{\operatorname{ar}\left(D_{\rho}\right)} \int_{D_{\rho}}(|\Lambda-\bar{\Lambda}|+\operatorname{dist}(\Lambda, S O(3)))^{2} \\
& =\frac{1}{\operatorname{ar}\left(D_{\rho}\right)}\|\Lambda-\bar{\Lambda}\|_{L^{2}\left(D_{\rho}\right)}^{2} .
\end{aligned}
$$

Thus, there exists a map $R \in S O(3)$ such that

$$
\|\Lambda-R\|_{L^{2}\left(D_{\rho}\right)} \leq \sqrt{2} C \rho\|d \Lambda\|_{L^{2}(D \rho)} .
$$

empty

\section{Acknowledgments}

We wish to thank Gerhard Huisken for bringing this problem to our attention and Eberhard Zeidler for suggesting the use of Cartan formalism in Section 'i ${ }_{-1}^{i}$. We also thank Daniel Faraco and Ernst Kuwert for interesting discussions. Both authors acknowledge partial support by the EU Network Hyperbolic and kinetic equations HPRN-CT-200200282 .

\section{References}

[1] W. Chen \& C. Li, Classification of solutions of some non-linear elliptic equations, Duke Math. J. 63 (1991) 615-623, MR 1121147, Zbl 10768.35025!.

[2] R. Coifman, P.L. Lions, Y. Meyer, \& S. Semmes, Compensated compactness and Hardy spaces, J. Math. Pures Appl. 72 (1993) 247-286, MR 122551]. Zbl 0864.42009

[3] C. De Lellis \& S. Müller, $A C^{0}$ estimate for nearly umbilical surfaces, in preparation.

[4] M.P. Do Carmo, Differential geometry of curves and surfaces, Prentice Hall, 1976, MR 0394451', Zbl 0326.53001!.

[5] M.P. Do_Carmo, Riemannian geometry, Birkhäuser, 1992, MR 1138207, Zbl 0752.53001

[6] C. Fefferman \& E.M. Stein, $\mathcal{H}^{p}$ spaces_of_several variables, Acta Math. 129 (1972) 137-193, MR p447953, Zbl 0257.46078.

[7] G. Huisken \& S.-T. Yau, Definition of center of mass for isolated physical systems and unique foliations by stable spheres with constant mean curvature, Invent. Math. 124 (1996) 281-311, MR 1369419, Zbl 0858.53071.

[8] E. Kuwert \& R. Schätzle, The Willmore flow with small initial energy, J. Differential Geom. 57 (2001) 409-441, MR 1882663, Zbl 1035.53092.

[9] Ch.B. Morrey, Multiple integrals in the calculus of variations, Springer, 1966, MR 0202511, Zbl 0142.38701 . 
[10] S. Müller \& V. Šverák, On surfaces of finite total_ curvature, J. Differential Geom. 42(2) (1995) 229-258, MR 1366547, Zbl 0853.53003!

[11] A.V. Pogorelov, Extrinsic geometry of convex_surfaces, Translations of Mathematical Monographs, 35, AMS, 1973, MR 0346714, Zbl 0311.53067.

[12] Y.G. Reshetnyak, Stability theorems in geometry and analysis, Mathematics and its Applications, 304,_Kluwer Academic Publishers Group, Dordrecht, 1994, MR 1326375i, Zbl 10848.30013

[13] E.H. Spanier, Algebraic topology, McGraw-Hill Book Co., 1966, MR 0210112 , $\mathrm{Zbl} 0145.43303$

[14] M. Spivak, A comprehensive introduction to differential geometry, Publish or Perish, Houston, 1979, MR 0532830 , Zbl

[15] E. Stein,_Harmonic Analysis, Princeton University Press, 1993, MR 1232192, $\mathrm{Zbl} 0821.42001 !$

[16] S.K. Vodop'yanov, Estimates for the deviation of quasi-umbilical surfaces from the sphere, Sibirsk. Math. Zh. 11(5) (1970) 971-987, MR 10298603, $\mathrm{Zbl}$, 214.49205

[17] W.P. Ziemer, Weakly differentiable functions, Springer, 1989, MR 1014685, $\mathrm{Zbl}$ 0692.46022

Max-Planck Institute for Mathematics in the Sciences INSELSTR. 22, D-04103 LEIPZIG

GERMany

E-mail address: delellis@mis.mpg.de

MaX-Planck Institute for Mathematics in the Sciences INSELSTR. 22, D-04103 LEIPZIG

GERMANy

E-mail address: sm@mis.mpg.de 